\title{
Kannan nonexpansive maps on generalized Cesàro backward difference sequence space of non-absolute type with applications to summable equations
}

\author{
Awad A. Bakery ${ }^{1,2^{*}}$ (D) and Om Kalthum S.K. Mohamed ${ }^{1,3}$
}

"Correspondence:
aabhassan@uj.edu.sa;
awad_bakery@yahoo.com;
awad_bakry@hotmail.com
'Department of Mathematics,
College of Science and Arts at
Khulis, University of Jeddah, Jeddah,
Saudi Arabia
2Department of Mathematics,
Faculty of Science, Ain Shams
University, P.O. Box 1156, Cairo,
11566, Abbassia, Egypt
Full list of author information is
available at the end of the article

available at the end of the article

\begin{abstract}
In this article, we investigate the notion of the pre-quasi norm on a generalized Cesàro backward difference sequence space of non-absolute type $(\boldsymbol{\Xi}(\Delta, r))_{\psi}$ under definite function $\psi$. We introduce the sufficient set-up on it to form a pre-quasi Banach and a closed special space of sequences (sss), the actuality of a fixed point of a Kannan pre-quasi norm contraction mapping on $(\boldsymbol{\Xi}(\Delta, r))_{\psi}$, it supports the property (R) and has the pre-quasi normal structure property. The existence of a fixed point of the Kannan pre-quasi norm nonexpansive mapping on $(\boldsymbol{\Xi}(\Delta, r))_{\psi}$ and the Kannan pre-quasi norm contraction mapping in the pre-quasi Banach operator ideal constructed by $(\boldsymbol{\Xi}(\Delta, r))_{\psi}$ and $s$-numbers has been determined. Finally, we support our results by some applications to the existence of solutions of summable equations and illustrative examples.
\end{abstract}

MSC: 46C05; 46B10; 46B15; 46E05; 46E15; 46E30; 47H09; 47H10

Keywords: Pre-quasi norm; Operator ideal; Property (R); Cesàro sequence space; Kannan contraction mapping; Kannan nonexpansive mapping; Electrorheological fluids

\section{Introduction}

Ideal operator theorems are very important in mathematical models and have numerous implementations, such as normal series theory, ideal transformations, geometry of Banach spaces, approximation theory, fixed point theory, and so forth. Nakano sequence spaces $\ell(r)$ are contained in the variable exponent spaces $L_{(r)}$. Regarding the second half of the twentieth century, it used to be fulfilled that these variable exponent spaces constituted the proper framework for the mathematical components of numerous issues for which the classical Lebesgue spaces have been inadequate. The relevancy of these spaces and their effects made them a famous and environment friendly device in the remedy of a range of situations. These days the region of $L_{(r)}(\Omega)$ spaces is a prolific subject of lookup with ramifications achieving into very numerous mathematical specialties [1]. Learning about the variable exponent Lebesgue spaces $L_{(r)}$ gained in addition impetus from the mathe-

(c) The Author(s) 2021. This article is licensed under a Creative Commons Attribution 4.0 International License, which permits use sharing, adaptation, distribution and reproduction in any medium or format, as long as you give appropriate credit to the original author(s) and the source, provide a link to the Creative Commons licence, and indicate if changes were made. The images or other third party material in this article are included in the article's Creative Commons licence, unless indicated otherwise in a credit line to the material. If material is not included in the article's Creative Commons licence and your intended use is not permitted by statutory regulation or exceeds the permitted use, you will need to obtain permission directly from the copyright holder. To view a copy of this licence, visit http://creativecommons.org/licenses/by/4.0/. 
matical description of the hydrodynamics of non-Newtonian fluids [2, 3]. Applications of non-Newtonian fluids, additionally known as electrorheological, vary from their use in army science to civil engineering and orthopedics. By $\mathfrak{R}^{\mathcal{N}}, \ell_{\infty}, \ell_{r}$, and $c_{0}$ we signify the spaces of each bounded, $r$-absolutely summable, and convergent to zero sequences of real numbers. We signify the space of all bounded linear operators from a Banach space $Z$ into a Banach space $M$ by $\mathcal{L}(Z, M)$, and if $Z=M$, we write $\mathcal{L}(Z)$, and $e_{d}=\{0,0, \ldots, 1,0,0, \ldots\}$, while 1 presents the $d^{\text {th }}$ place for every $d \in \mathcal{N}=\{0,1,2, \ldots\}$.

Definition 1.1 ([4]) An $s$-number function is a map defined on $\mathcal{L}(Z, M)$ which sort to every map $W \in \mathcal{L}(Z, M)$ a nonnegative scaler sequence $\left(s_{d}(W)\right)_{d=0}^{\infty}$ satisfies the following setting:

(a) $\|W\|=s_{0}(W) \geq s_{1}(W) \geq s_{2}(W) \geq \cdots \geq 0$ for all $W \in \mathcal{L}(Z, M)$;

(b) $s_{l+d}\left(W_{1}+W_{2}\right) \leq s_{l}\left(W_{1}\right)+s_{d}\left(W_{2}\right)$ for every $W_{1}, W_{2} \in \mathcal{L}(Z, M)$ and $l, d \in \mathcal{N}$;

(c) Ideal property: $s_{d}(V Y W) \leq\|V\| s_{d}(Y)\|W\|$ for every $W \in \mathcal{L}\left(Z_{0}, Z\right), Y \in \mathcal{L}(Z, M)$ and $V \in \mathcal{L}\left(M, M_{0}\right)$, where $Z_{0}$ and $M_{0}$ are discretionary Banach spaces;

(d) For $W \in \mathcal{L}(Z, M)$ and $\gamma \in \Re$, one has $s_{d}(\gamma W)=|\gamma| s_{d}(W)$;

(e) Rank property: Assume $\operatorname{rank}(W) \leq d$, then $s_{d}(W)=0$ for each $W \in \mathcal{L}(Z, M)$;

(f) Norming property: $s_{l \geq a}\left(I_{a}\right)=0$ or $s_{l<a}\left(I_{a}\right)=1$, where $I_{a}$ mirrors the unit map on the $a$-dimensional Hilbert space $\ell_{2}^{a}$.

The $d$ th approximation number, established by $\alpha_{d}(W)$, is defined as follows:

$$
\alpha_{d}(W)=\inf \{\|W-Y\|: Y \in \mathcal{L}(Z, M) \text { and } \operatorname{rank}(Y) \leq d\}
$$

Notations 1.2 The sets $S_{A}, S_{A}(Z, M), S_{A}^{\text {app }}$, and $S_{A}^{\text {app }}(Z, M)$ (cf. [5]) are as follows:

$$
S_{A}:=\left\{S_{A}(Z, M)\right\}, \quad \text { where } S_{A}(Z, M):=\left\{W \in \mathcal{L}(Z, M):\left(\left(s_{d}(W)\right)_{d=0}^{\infty} \in A\right\} .\right.
$$

Also

$$
S_{A}^{\mathrm{app}}:=\left\{S_{A}^{\mathrm{app}}(Z, M)\right\}, \quad \text { where } S_{A}^{\mathrm{app}}(Z, M):=\left\{W \in \mathcal{L}(Z, M):\left(\left(\alpha_{d}(W)\right)_{d=0}^{\infty} \in A\right\} .\right.
$$

In [5], Faried and Bakery made known the theory of pre-quasi operator ideal that is more conventional than the quasi operator ideal. Bakery and Abou Elmatty [6] gave the sufficient (not necessary) conditions on $\ell(r)$ such that $S_{\ell(r)}$ constructed a simple Banach pre-quasi operator ideal. The pre-quasi operator ideal $S_{\ell(r)}^{\text {app }}$ was once strictly contained for different exponents. It was once a small pre-quasi operator ideal. Since the booklet of the Banach fixed point theorem [7], many mathematicians worked on feasible extensions. Kannan [8] approved an instance of a class of operators with the identical fixed point actions as contractions though that flop to be continuous. The only attempt to describe Kannan operators in modular vector spaces was made by Ghoncheh [9]. The intention of this paper is to learn about the thinking of pre-quasi norm on $\Xi(\Delta, r)$. We explain the sufficient conditions on $\Xi(\Delta, r)$ equipped with the definite pre-quasi norm to structure pre-quasi Banach and closed (sss), the existence of a fixed point of a Kannan pre-quasi norm contraction mapping in the pre-quasi Banach (sss), $(\Xi(\Delta, r))_{\psi}$ satisfies the property (R) and $(\Xi(\Delta, r))_{\psi}$ has the $\psi$-normal structure property. The existence of a fixed point of 
the Kannan pre-quasi norm nonexpansive mapping in the pre-quasi Banach (sss) is determined. In addition, we provide the idea of a Kannan pre-quasi norm contraction mapping in the pre-quasi operator ideal. The existence of a fixed point of the Kannan pre-quasi norm contraction mapping in the pre-quasi Banach operator ideal $S_{(\Xi(\Delta, r))_{\psi}}$ is given. Finally, we give some applications to the existence of solutions of summable equations with some examples to illustrate our results.

\section{Definitions and preliminaries}

Definition 2.1 ([5]) The linear space of sequences $\mathfrak{A}$ is known as a special space of sequences (sss) if:

(1) $\left\{e_{a}\right\}_{a \in \mathcal{N}} \subseteq \mathfrak{A}$;

(2) $\mathfrak{A}$ is solid, i.e., conceited $v=\left(v_{a}\right) \in \mathfrak{R}^{\mathcal{N}}, t=\left(t_{a}\right) \in \mathfrak{A}$, and $\left|v_{a}\right| \leq\left|t_{a}\right|$ for each $a \in \mathcal{N}$, then $v \in \mathfrak{A}$;

(3) $\left(v_{\left[\frac{a}{2}\right]}\right]_{a=0}^{\infty} \in \mathfrak{A}$, where $\left[\frac{a}{2}\right]$ illustrates the integral part of $\frac{a}{2}$ in case $\left(v_{a}\right)_{a=0}^{\infty} \in \mathfrak{A}$.

By $[0, \infty)^{\mathfrak{A}}$, we denote the space of all functions $\phi: \mathfrak{A} \longrightarrow[0, \infty)$.

Definition 2.2 ([5]) A subclass $\mathfrak{A}_{\psi}$ of $\mathfrak{A}$ is called a pre-modular (sss) if there is $\psi \in[0, \infty)^{\mathfrak{A}}$ with the following:

(i) For $v \in \mathfrak{A}, v=\theta \Leftrightarrow \psi(v)=0$ with $\psi(v) \geq 0$, where $\theta$ is the zero vector of $\mathfrak{A}$;

(ii) For all $v \in \mathfrak{A}$ and $\eta \in \mathfrak{R}$, there is $B \geq 1$ for which $\psi(\eta v) \leq B|\eta| \psi(v)$;

(iii) $\psi(v+t) \leq J(\psi(v)+\psi(t))$ for each $v, t \in \mathfrak{A}$ embodies for some $J \geq 1$;

(iv) For $a \in \mathcal{N}$ and $\left|v_{a}\right| \leq\left|t_{a}\right|$, we obtain $\psi\left(\left(v_{a}\right)\right) \leq \psi\left(\left(t_{a}\right)\right)$;

(v) The inequality $\psi\left(\left(v_{a}\right)\right) \leq \psi\left(\left(v_{\left[\frac{a}{2}\right]}\right)\right) \leq J_{0} \psi\left(\left(v_{a}\right)\right)$ holds for some $J_{0} \geq 1$;

(vi) If $F$ is the space of finite sequences, then $\bar{F}=\mathfrak{A}_{\psi}$;

(vii) There is $\varsigma>0$ such that $\psi(\beta, 0,0,0, \ldots) \geq \varsigma|\beta| \psi(1,0,0,0, \ldots)$ for every $\beta \in \mathfrak{R}$.

Definition 2.3 ([10]) Let $\mathfrak{A}$ be a (sss). The function $\psi \in[0, \infty)^{\mathfrak{A}}$ is named a pre-quasi norm on $\mathfrak{A}$ if it provides the following setting:

(i) For $v \in \mathfrak{A}, v=\theta \Leftrightarrow \psi(v)=0$ with $\psi(v) \geq 0$, where $\theta$ is the zero vector of $\mathfrak{A}$;

(ii) For some $B \geq 1$, the inequality $\psi(\eta v) \leq B|\eta| \psi(v)$ holds for all $v \in \mathfrak{A}$ and $\eta \in \mathfrak{R}$;

(iii) For some $J \geq 1$, the inequality $\psi(v+t) \leq J(\psi(v)+\psi(t))$ is satisfied for all $v, t \in \mathfrak{A}$.

Theorem 2.4 ([10]) If $\mathfrak{A}$ is a pre-modular (sss), then it is a pre-quasi normed (sss).

Theorem 2.5 ([10]) $\mathfrak{A}$ is a pre-quasi normed (sss) if it is a quasi-normed (sss).

Definition 2.6 ([11]) If $\mathcal{L}$ is the class of bounded linear maps within any two Banach spaces. A subclass $\mathcal{U}$ of $\mathcal{L}$ is named an operator ideal if every element $\mathcal{U}(Z, M)=\mathcal{U} \cap$ $\mathcal{L}(Z, M)$ fulfills the following setting:

(i) $I_{\Gamma} \in \mathcal{U}$ throughout $\Gamma$ characterizes a Banach space of one dimension.

(ii) The space $\mathcal{U}(Z, M)$ is linear over $\mathfrak{R}$.

(iii) If $W \in \mathcal{L}\left(Z_{0}, Z\right), X \in \mathcal{U}(Z, M)$, and $Y \in \mathcal{L}\left(M, M_{0}\right)$, then $Y X W \in \mathcal{U}\left(Z_{0}, M_{0}\right)$, where $Z_{0}$ and $M_{0}$ are normed spaces.

The notion of pre-quasi operator ideal is more regular than the quasi operator ideal. 
Definition 2.7 ([5]) A function $\Psi \in[0, \infty)^{\mathcal{U}}$ is named a pre-quasi norm on the ideal $\mathcal{U}$ if the following setting holds:

(1) Assume $W \in \mathcal{U}(Z, M), \Psi(W) \geq 0$, and $\Psi(W)=0$ if and only if $W=0$;

(2) There is $D \geq 1$ so as to $\Psi(\eta W) \leq D|\eta| \Psi(W)$ for all $W \in \mathcal{U}(Z, M)$ and $\eta \in \Re$;

(3) There is $J \geq 1$ so that $\Psi\left(W_{1}+W_{2}\right) \leq J\left[\Psi\left(W_{1}\right)+\Psi\left(W_{2}\right)\right]$ for all $W_{1}, W_{2} \in \mathcal{U}(Z, M)$;

(4) There is $\sigma \geq 1$ if $W \in \mathcal{L}\left(Z_{0}, Z\right), X \in \mathcal{U}(Z, M)$, and $Y \in \mathcal{L}\left(M, M_{0}\right)$, then $\Psi(Y X W) \leq \sigma\|Y\| \Psi(X)\|W\|$.

Theorem 2.8 ([12]) Pick up $\mathfrak{A}_{\psi}$ to be a pre-modular (sss), then $\Psi(W)=\psi\left(s_{a}(W)\right)_{a=0}^{\infty}$ is a pre-quasi norm on $S_{\mathfrak{A}_{\psi}}$.

Theorem 2.9 ([6]) Let $Z$ and $M$ be Banach spaces and $\mathfrak{A}_{\psi}$ be a pre-modular (sss), then $\left(S_{\mathfrak{A}_{\psi}}, \Psi\right)$ is a pre-quasi Banach operator ideal, so that $\Psi(W)=\psi\left(\left(s_{a}(W)\right)_{a=0}^{\infty}\right)$.

Theorem $2.10([5]) \Psi$ is a pre-quasi norm on the ideal $\mathcal{U}$ if $\Psi$ is a quasi norm on the ideal $\mathcal{U}$.

Lemma 2.11 The given inequalities will be used in the sequel:

(i) [13] Let $r \geq 2$ and for every $v, t \in \mathfrak{R}$, then

$$
\left|\frac{v+t}{2}\right|^{r}+\left|\frac{v-t}{2}\right|^{r} \leq \frac{1}{2}\left(|v|^{r}+|t|^{r}\right) .
$$

(ii) [14] Assume $1<r \leq 2$ and for all $v, t \in \mathfrak{R}$ so that $|v|+|t| \neq 0$, then

$$
\left|\frac{v+t}{2}\right|^{r}+\frac{r(r-1)}{2}\left|\frac{v-t}{|v|+|t|}\right|^{2-r}\left|\frac{v-t}{2}\right|^{r} \leq \frac{1}{2}\left(|v|^{r}+|t|^{r}\right) .
$$

(iii) [15] Suppose $r_{a}>0$ and $v_{a}, t_{a} \in \mathfrak{R}$ for every $a \in \mathcal{N}$, then $\left|v_{a}+t_{a}\right|^{r_{a}} \leq 2^{K-1}\left(\left|v_{a}\right|^{r_{a}}+\left|t_{a}\right|^{r_{a}}\right)$, where $K=\max \left\{1, \sup _{a} r_{a}\right\}$.

\section{The sequence space $(\Xi(\Delta, r))_{\psi}$}

We introduce in this section the definition of generalized Cesàro backward difference sequence space of non-absolute type $(\Xi(\Delta, r))_{\psi}$ under the function $\psi$ and some inclusion relations.

The Cesàro matrix $\Lambda_{1}$ of order 1 is represented explicitly as

$$
\Lambda_{1}=\left[\begin{array}{ccccc}
1 & 0 & 0 & 0 & \ldots \\
\frac{1}{2} & \frac{1}{2} & 0 & 0 & \ldots \\
\frac{1}{3} & \frac{1}{3} & \frac{1}{3} & 0 & \ldots \\
\frac{1}{4} & \frac{1}{4} & \frac{1}{4} & \frac{1}{4} & \ldots \\
\vdots & \vdots & \vdots & \vdots & \ddots
\end{array}\right]
$$

Definition 3.1 For all $\left(r_{l}\right) \in \mathfrak{R}^{+\mathcal{N}}$, the sequence spaces $\left(\ell_{\infty}(r)\right)_{\psi},\left(c_{0}(r)\right)_{\psi}$, and $(\ell(r))_{\psi}$ are defined as follows:

$$
\left(\ell_{\infty}(r)\right)_{\psi_{\infty}}=\left\{f=\left(f_{k}\right) \in \mathfrak{R}^{\mathcal{N}}: \psi_{\infty}(\rho f)<\infty \text { for some } \rho>0\right\}
$$


where $\psi_{\infty}(f)=\sup _{l}\left|f_{l}\right|^{r_{l}}$.

$\left(c_{0}(r)\right)_{\psi_{\infty}}=\left\{f=\left(f_{k}\right) \in \mathfrak{R}^{\mathcal{N}}: \lim _{l \rightarrow \infty}\left|\rho f_{l}\right|^{r_{l}}=0\right.$ for some $\left.\rho>0\right\}$,

where $\psi_{\infty}(f)=\sup _{l}\left|f_{l}\right|^{r_{l}}$.

$(\ell(r))_{\psi}=\left\{f=\left(f_{k}\right) \in \mathfrak{R}^{\mathcal{N}}: \psi(\rho f)<\infty\right.$ for some $\left.\rho>0\right\}$,

where $\psi(f)=\sum_{l=0}^{\infty}\left|f_{l}\right|^{r_{l}}$.

Definition 3.2 For all $\left(r_{l}\right) \in \mathfrak{R}^{+\mathcal{N}}$, we define the following sequence spaces:

$$
\begin{aligned}
& \Xi_{\infty}(\Delta, r)=\left\{f=\left(f_{k}\right) \in \mathfrak{R}^{\mathcal{N}}: \Lambda_{1} \Delta f \in \ell_{\infty}(r)\right\} . \\
& \Xi_{0}(\Delta, r)=\left\{f=\left(f_{k}\right) \in \mathfrak{R}^{\mathcal{N}}: \Lambda_{1} \Delta f \in c_{0}(r)\right\} . \\
& \Xi(\Delta, r)=\left\{f=\left(f_{k}\right) \in \mathfrak{R}^{\mathcal{N}}: \Lambda_{1} \Delta f \in \ell(r)\right\} .
\end{aligned}
$$

Theorem 3.3 If $\left(r_{l}\right) \in \mathfrak{R}^{+\mathcal{N}}$, then $\Xi(\Delta, r) \varsubsetneqq \Xi_{0}(\Delta, r) \varsubsetneqq \Xi_{\infty}(\Delta, r)$.

Definition 3.4 For all $\left(r_{l}\right) \in \mathfrak{R}^{+\mathcal{N}}$, the sequence space $(\Xi(\Delta, r))_{\psi}$ under the function $\psi$ is defined as follows:

$$
(\Xi(\Delta, r))_{\psi}=\left\{f=\left(f_{k}\right) \in \mathfrak{R}^{\mathcal{N}}: \psi(\rho f)<\infty \text { for some } \rho>0\right\},
$$

where $\psi(f)=\sum_{l=0}^{\infty}\left(\frac{\left|\sum_{z=0}^{l} \Delta f_{z}\right|}{l+1}\right)^{r} l$ and $\Delta f_{z}=f_{z}-f_{z-1}$ with $f_{z}=0$ for $z<0$.

Theorem 3.5 If $\left(r_{l}\right) \in \mathfrak{R}^{+\mathcal{N}} \cap \ell_{\infty}$, then

$$
(\Xi(\Delta, r))_{\psi}=\left\{f=\left(f_{k}\right) \in \mathfrak{R}^{\mathcal{N}}: \psi(\rho f)<\infty \text { for any } \rho>0\right\} .
$$

Proof Assume $\left(r_{l}\right) \in \mathfrak{R}^{+\mathcal{N}} \cap \ell_{\infty}$, one has

$$
\begin{aligned}
(\Xi(\Delta, r))_{\psi} & =\left\{f=\left(f_{k}\right) \in \mathfrak{R}^{\mathcal{N}}: \psi(\rho f)<\infty \text { for some } \rho>0\right\} \\
& =\left\{f=\left(f_{k}\right) \in \mathfrak{R}^{\mathcal{N}}: \sum_{l=0}^{\infty}\left(\frac{\left|\sum_{z=0}^{l} \Delta \rho f_{z}\right|}{l+1}\right)^{r_{l}}<\infty \text { for some } \rho>0\right\} \\
& =\left\{f=\left(f_{k}\right) \in \mathfrak{R}^{\mathcal{N}}: \inf _{l} \rho^{r_{l}} \sum_{l=0}^{\infty}\left(\frac{\left|\sum_{z=0}^{l} \Delta f_{z}\right|}{l+1}\right)^{r_{l}}<\infty \text { for some } \rho>0\right\} \\
& =\left\{f=\left(f_{k}\right) \in \mathfrak{R}^{\mathcal{N}}: \sum_{l=0}^{\infty}\left(\frac{\left|\sum_{z=0}^{l} \Delta f_{z}\right|}{l+1}\right)^{r_{l}}<\infty\right\} \\
& =\left\{f=\left(f_{k}\right) \in \mathfrak{R}^{\mathcal{N}}: \psi(\rho f)<\infty \text { for any } \rho>0\right\} .
\end{aligned}
$$

Theorem 3.6 If $\left(r_{l}\right) \in \mathfrak{R}^{+\mathcal{N}} \cap \ell_{\infty}$, then $(\Xi(\Delta, r))_{\psi}$ is of absolute type. 
Proof We have

$$
\psi(f)=\sum_{l=0}^{\infty}\left(\frac{\left|\sum_{z=0}^{l} \Delta f_{z}\right|}{l+1}\right)^{r_{l}}=\sum_{l=0}^{\infty}\left(\frac{\left|f_{l}\right|}{l+1}\right)^{r_{l}}=\sum_{l=0}^{\infty}\left(\frac{\left|\sum_{z=0}^{l} \Delta\right| f_{z}||}{l+1}\right)^{r_{l}}=\psi(|f|) .
$$

Therefore, the sequence space $(\Xi(\Delta, r))_{\psi}$ is of absolute type.

Theorem 3.7 If $\left(r_{l}\right) \in \mathfrak{R}^{+\mathcal{N}} \cap \ell_{\infty}$, then $(\ell(r))_{\psi} \varsubsetneqq(\Xi(\Delta, r))_{\psi}$.

Proof Let $f \in(\ell(r))_{\psi}$, since

$$
\sum_{l=0}^{\infty}\left(\frac{\left|\sum_{z=0}^{l} \Delta f_{z}\right|}{l+1}\right)^{r_{l}}=\sum_{l=0}^{\infty}\left(\frac{\left|f_{l}\right|}{l+1}\right)^{r_{l}} \leq \sum_{l=0}^{\infty}\left|f_{l}\right|^{r_{l}}<\infty,
$$

then $f \in(\Xi(\Delta, r))_{\psi}$. For $\left(r_{l}\right) \in(1, \infty)^{\mathcal{N}} \cap \ell_{\infty}$, we choose $f=(1,1,1, \ldots)$, one has $f \in$ $(\Xi(\Delta, r))_{\psi}$ and $f \notin \ell(r)$. For $\left(r_{l}\right) \in(0,1]^{\mathcal{N}}$, we choose $f=\left(\frac{1}{l+1}\right)^{r_{l}^{-1}}$, one has $f \in(\Xi(\Delta, r))_{\psi}$ and $f \notin \ell(r)$.

Definition 3.8 For all $\left(r_{l}\right) \in \mathfrak{R}^{+\mathcal{N}} \cap \ell_{\infty}$, the generalized Cesàro backward difference sequence space of absolute type $(\operatorname{ces}(\Delta, r))_{\varphi}$ is defined as follows:

$$
\begin{aligned}
& (\operatorname{ces}(\Delta, r))_{\varphi}=\left\{f=\left(f_{k}\right) \in \mathfrak{R}^{\mathcal{N}}: \varphi(\rho f)<\infty \text { for some } \rho>0\right\} \\
& \text { where } \varphi(f)=\sum_{l=0}^{\infty}\left(\frac{\sum_{z=0}^{l}\left|\Delta f_{z}\right|}{l+1}\right)^{r_{l}} .
\end{aligned}
$$

Theorem 3.9 If $\left(r_{l}\right) \in \mathfrak{R}^{+\mathcal{N}} \cap \ell_{\infty}$, then $(\operatorname{ces}(\Delta, r))_{\varphi} \varsubsetneqq(\Xi(\Delta, r))_{\psi}$.

Proof Let $f \in(\operatorname{ces}(\Delta, r))_{\varphi}$, since

$$
\sum_{l=0}^{\infty}\left(\frac{\left|\sum_{z=0}^{l} \Delta f_{z}\right|}{l+1}\right)^{r_{l}} \leq \sum_{l=0}^{\infty}\left(\frac{\sum_{z=0}^{l}\left|\Delta f_{z}\right|}{l+1}\right)^{r_{l}}<\infty .
$$

Then $f \in(\Xi(\Delta, r))_{\psi}$. For $\left(r_{l}\right) \in(1, \infty)^{\mathcal{N}} \cap \ell_{\infty}$, we choose $f=\left((-1)^{z}\right)_{z \in \mathcal{N}}$, one has $f \in$ $(\Xi(\Delta, r))_{\psi}$ and $f \notin(\operatorname{ces}(\Delta, r))_{\varphi}$. For $\left(r_{l}\right) \in(0,1]^{\mathcal{N}}$, we choose $f=(1,0,0, \ldots)$, one has $f \in$ $(\Xi(\Delta, r))_{\psi}$ and $f \notin(\operatorname{ces}(\Delta, r))_{\varphi}$.

\section{Pre-quasi norm on $\Xi(\Delta, r)$}

We investigate in this section the sufficient set-up on $\Xi(\Delta, r)$ with a pre-quasi norm $\psi$ to form a pre-quasi Banach and a closed (sss). The Fatou property for different pre-quasi norm $\psi$ on $\Xi(\Delta, r)$ is studied.

\section{Definition 4.1}

(a) The function $\psi$ on $\Xi(\Delta, r)$ is called $\psi$-convex if

$$
\psi(\omega v+(1-\omega) t) \leq \omega \psi(v)+(1-\omega) \psi(t)
$$

for each $\omega \in[0,1]$ and $v, t \in \Xi(\Delta, r)$. 
(b) $\left\{v_{a}\right\}_{a \in \mathcal{N}} \subseteq(\Xi(\Delta, r))_{\psi}$ is $\psi$-convergent to $v \in(\Xi(\Delta, r))_{\psi}$ if and only if $\lim _{a \rightarrow \infty} \psi\left(v_{a}-v\right)=0$. If the $\psi$-limit exists, then it is unique.

(c) $\left\{v_{a}\right\}_{a \in \mathcal{N}} \subseteq(\Xi(\Delta, r))_{\psi}$ is $\psi$-Cauchy, when $\lim _{a, b \rightarrow \infty} \psi\left(v_{a}-v_{b}\right)=0$.

(d) $\Lambda \subset(\Xi(\Delta, r))_{\psi}$ is $\psi$-closed, if for all $\psi$-converging $\left\{v_{a}\right\}_{a \in \mathcal{N}} \subset \Lambda$ to $v$, then $v \in \Lambda$.

(e) $\Lambda \subset(\Xi(\Delta, r))_{\psi}$ is $\psi$-bounded, when $\delta_{\psi}(\Lambda)=\sup \{\psi(v-t): v, t \in \Lambda\}<\infty$.

(f) The $\psi$-ball of radius $d \geq 0$ and center $v$, for every $v \in(\Xi(\Delta, r))_{\psi}$, is detailed

$$
\mathcal{B}_{\psi}(v, d)=\left\{t \in(\Xi(\Delta, r))_{\psi}: \psi(v-t) \leq d\right\} .
$$

(g) A pre-quasi norm $\psi$ on $\Xi(\Delta, r)$ satisfies the Fatou property if for any sequence $\left\{t^{a}\right\} \subseteq(\Xi(\Delta, r))_{\psi}$ with $\lim _{a \rightarrow \infty} \psi\left(t^{a}-t\right)=0$ and any $v \in(\Xi(\Delta, r))_{\psi}$, then $\psi(v-t) \leq \sup _{j} \inf _{a \geq j} \psi\left(v-t^{a}\right)$.

Note that the Fatou property gives the $\psi$-closedness of the $\psi$-balls.

Theorem 4.2 If $\left(r_{a}\right) \in \mathfrak{R}^{+\mathcal{N}} \cap \ell_{\infty}$ is increasing, then the space $(\Xi(\Delta, r))_{\psi}$ is a pre-modular $($ sss $)$, where $\psi(v)=\left[\sum_{a=0}^{\infty}\left(\frac{\left|\sum_{z=0}^{a} \Delta v_{z}\right|}{a+1}\right)^{r}\right]^{\frac{1}{K}}$ for each $v \in \Xi(\Delta, r)$.

Proof Firstly, we have to prove $\Xi(\Delta, r)$ is a (sss):

(1-i) Suppose $v, t \in \Xi(\Delta, r)$. Since $\left(r_{a}\right)$ is bounded, we obtain

$$
\begin{aligned}
\psi(v+t) & =\left[\sum_{a=0}^{\infty}\left(\frac{\left|v_{a}+t_{a}\right|}{a+1}\right)^{r_{a}}\right]^{\frac{1}{K}} \leq\left[\sum_{a=0}^{\infty}\left(\frac{\left|v_{a}\right|}{a+1}\right)^{r_{a}}\right]^{\frac{1}{K}}+\left[\sum_{a=0}^{\infty}\left(\frac{\left|t_{a}\right|}{a+1}\right)^{r_{a}}\right]^{\frac{1}{K}} \\
& =\psi(v)+\psi(t)<\infty,
\end{aligned}
$$

so $v+t \in \Xi(\Delta, r)$.

(1-ii) Assume $\eta \in \mathfrak{R}$ and $v \in \Xi(\Delta, r)$. As $\left(r_{a}\right)$ is bounded, we have

$$
\psi(\eta v)=\left[\sum_{a=0}^{\infty}\left(\frac{\left|\eta v_{a}\right|}{a+1}\right)^{r_{a}}\right]^{\frac{1}{K}} \leq \sup _{a}|\eta|^{r_{a}}\left[\sum_{a=0}^{\infty}\left(\frac{\left|v_{a}\right|}{a+1}\right)^{r_{a}}\right]^{\frac{1}{K}} \leq D|\eta| \psi(v)<\infty .
$$

Hence $\eta v \in \Xi(\Delta, r)$. Then, by using Parts (1-i) and (1-ii), we get $\Xi(\Delta, r)$ is a linear space. Also $e_{a} \in \Xi(\Delta, r)$ for all $a \in \mathcal{N}$ since

$$
\psi\left(e_{a}\right)=\left[\sum_{j=0}^{\infty}\left(\frac{\left|e_{a}(j)\right|}{j+1}\right)^{r_{j}}\right]^{\frac{1}{K}}=\left(\frac{1}{a+1}\right)^{\frac{r_{a}}{K}} .
$$

(2) Let $\left|v_{a}\right| \leq\left|t_{a}\right|$ for all $a \in \mathcal{N}$ and $t \in \Xi(\Delta, r)$. One has

$$
\psi(v)=\left[\sum_{a=0}^{\infty}\left(\frac{\left|v_{a}\right|}{a+1}\right)^{r_{a}}\right]^{\frac{1}{K}} \leq\left[\sum_{a=0}^{\infty}\left(\frac{\left|t_{a}\right|}{a+1}\right)^{r_{a}}\right]^{\frac{1}{K}}=\psi(t)<\infty,
$$

we get $v \in \Xi(\Delta, r)$.

(3) Let $\left(v_{a}\right) \in \Xi(\Delta, r)$, one can see

$$
\psi\left(\left(v_{\left[\frac{a}{2}\right]}\right]\right)=\left[\sum_{a=0}^{\infty}\left(\frac{\left|v_{\left[\frac{a}{2}\right]}\right|}{a+1}\right)^{r_{a}}\right]^{\frac{1}{K}}=\left[\sum_{a=0}^{\infty}\left(\frac{\left|v_{a}\right|}{2 a+1}\right)^{r_{2 a}}+\sum_{a=0}^{\infty}\left(\frac{\left|v_{a}\right|}{2 a+2}\right)^{r_{2 a+1}}\right]^{\frac{1}{K}}
$$




$$
\leq 2^{\frac{1}{K}}\left[\sum_{a=0}^{\infty}\left(\frac{\left|v_{a}\right|}{a+1}\right)^{r_{a}}\right]^{\frac{1}{K}}=2^{\frac{1}{K}} \psi\left(\left(v_{a}\right)\right),
$$

then $\left(v_{\left[\frac{a}{2}\right]}\right) \in \Xi(\Delta, r)$. Secondly, we show that the function $\psi$ on $\Xi(\Delta, r)$ is pre-modular:

(i) Evidently, $\psi(v) \geq 0$ and $\psi(v)=0 \Leftrightarrow v=\theta$.

(ii) We have $D=\max \left\{1, \sup _{a}|\eta|^{\frac{r_{a}}{K}-1}\right\} \geq 1$ so that $\psi(\eta v) \leq D|\eta| \psi(v)$ for every $v \in \Xi(\Delta, r)$ and $\eta \in \mathfrak{R}$.

(iii) There is $J \geq 1$ such that $\psi(v+t) \leq J(\psi(v)+\psi(t))$ for all $v, t \in \Xi(\Delta, r)$.

(iv) Clearly from (2).

(v) It is obtained from (3) that $J_{0}=2^{\frac{1}{K}} \geq 1$.

(vi) Patently $\bar{F}=\Xi(\Delta, r)$.

(vii) There is $\varsigma$ so that $0<\varsigma \leq|\beta|^{\frac{r_{0}}{K}-1}$ for $\beta \neq 0$ or $\varsigma>0$, for $\beta=0$ such that

$$
\psi(\beta, 0,0,0, \ldots) \geq \varsigma|\beta| \psi(1,0,0,0, \ldots) .
$$

Theorem 4.3 If $\left(r_{a}\right) \in \mathfrak{R}^{+\mathcal{N}} \cap \ell_{\infty}$ is increasing, then $(\Xi(\Delta, r))_{\psi}$ is a pre-quasi Banach (sss), where $\psi(v)=\left[\sum_{a=0}^{\infty}\left(\frac{\left|\sum_{z=0}^{a} \Delta v_{z}\right|}{a+1}\right)^{r}\right]^{\frac{1}{K}}$ for each $v \in \Xi(\Delta, r)$.

Proof Assume that the set-up is verified. From Theorem 4.2, the space $(\Xi(\Delta, r))_{\psi}$ is a pre-modular (sss). By Theorem 2.4, the space $(\Xi(\Delta, r))_{\psi}$ is a pre-quasi normed (sss). To demonstrate that $(\Xi(\Delta, r))_{\psi}$ is a pre-quasi Banach (sss), assume $v^{p}=\left(v_{a}^{p}\right)_{a=0}^{\infty}$ to be a Cauchy sequence in $(\Xi(\Delta, r))_{\psi}$. Hence, for every $\varepsilon \in(0,1)$, there is $p_{0} \in \mathcal{N}$ so that, for all $p, q \geq p_{0}$, one has

$$
\psi\left(v^{p}-v^{q}\right)=\left[\sum_{a=0}^{\infty}\left(\frac{\left|v_{a}^{p}-v_{a}^{q}\right|}{a+1}\right)^{r_{a}}\right]^{\frac{1}{K}}<\varepsilon .
$$

Therefore, for $p, q \geq p_{0}$ and $a \in \mathcal{N}$, we obtain $\left|v_{a}^{p}-v_{a}^{q}\right|<\varepsilon$. So $\left(v_{a}^{q}\right)$ is a Cauchy sequence in $\mathfrak{R}$ for constant $a \in \mathcal{N}$, which implies $\lim _{q \rightarrow \infty} v_{a}^{q}=v_{a}^{0}$ for fixed $a \in \mathcal{N}$. Hence $\psi\left(v^{p}-v^{0}\right)<\varepsilon$ for each $p \geq p_{0}$. Conclusively to show that $v^{0} \in \Xi(\Delta, r)$, we have $\psi\left(v^{0}\right)=\psi\left(v^{0}-v^{p}+v^{p}\right) \leq$ $\psi\left(v^{p}-v^{0}\right)+\psi\left(v^{p}\right)<\infty$, so $v^{0} \in \Xi(\Delta, r)$. This means that $(\Xi(\Delta, r))_{\psi}$ is a pre-quasi Banach (sss).

Theorem 4.4 If $\left(r_{a}\right) \in \mathfrak{R}^{+\mathcal{N}} \cap \ell_{\infty}$ is increasing, then $(\Xi(\Delta, r))_{\psi}$ is a pre-quasi closed (sss), where $\psi(v)=\left[\sum_{a=0}^{\infty}\left(\frac{\left|\sum_{z=0}^{a} \Delta v_{z}\right|}{a+1}\right)^{r}\right]^{\frac{1}{K}}$ for each $v \in \Xi(\Delta, r)$.

Proof Assume that the set-up is verified. From Theorem 4.2, the space $(\Xi(\Delta, r))_{\psi}$ is a pre-modular (sss). By Theorem 2.4, the space $(\Xi(\Delta, r))_{\psi}$ is a pre-quasi normed (sss). To show that $(\Xi(\Delta, r))_{\psi}$ is a pre-quasi closed (sss), assume $v^{p}=\left(v_{a}^{p}\right)_{a=0}^{\infty} \in(\Xi(\Delta, r))_{\psi}$ and $\lim _{p \rightarrow \infty} \psi\left(v^{p}-v^{0}\right)=0$, then for every $\varepsilon \in(0,1)$, there is $p_{0} \in \mathcal{N}$ such that, for all $p \geq p_{0}$, one has

$$
\psi\left(v^{p}-v^{0}\right)=\left[\sum_{a=0}^{\infty}\left(\frac{\left|v_{a}^{p}-v_{a}^{0}\right|}{a+1}\right)^{r_{a}}\right]^{\frac{1}{K}}<\varepsilon .
$$

Hence, for $p \geq p_{0}$ and $a \in \mathcal{N}$, we get $\left|v_{a}^{p}-v_{a}^{0}\right|<\varepsilon$. So $\left(v_{a}^{p}\right)$ is a convergent sequence in $\mathfrak{R}$ for fixed $a \in \mathcal{N}$. Therefore, $\lim _{p \rightarrow \infty} v_{a}^{p}=v_{a}^{0}$ for fixed $a \in \mathcal{N}$. Finally, to prove that $v^{0} \in \Xi(\Delta, r)$, 
we have

$$
\psi\left(v^{0}\right)=\psi\left(v^{0}-v^{p}+v^{p}\right) \leq \psi\left(v^{p}-v^{0}\right)+\psi\left(v^{p}\right)<\infty,
$$

so $v^{0} \in \Xi(\Delta, r)$. This means that $(\Xi(\Delta, r))_{\psi}$ is a pre-quasi closed (sss).

Theorem 4.5 If $\left(r_{a}\right) \in \mathfrak{R}^{+\mathcal{N}} \cap \ell_{\infty}$ is increasing, then $\psi(v)=\left[\sum_{a=0}^{\infty}\left(\frac{\left|\sum_{z=0}^{a} \Delta v_{z}\right|}{a+1}\right)^{r_{a}}\right]^{\frac{1}{K}}$ verifies the Fatou property.

Proof Suppose that the set-up is fulfilled and $\left\{t^{b}\right\} \subseteq(\Xi(\Delta, r))_{\psi}$ with $\lim _{b \rightarrow \infty} \psi\left(t^{b}-t\right)=$ 0 . Since the space $(\Xi(\Delta, r))_{\psi}$ is pre-quasi closed, then $t \in(\Xi(\Delta, r))_{\psi}$. Then, for any $v \in$ $(\Xi(\Delta, r))_{\psi}$, one can see

$$
\begin{aligned}
\psi(v-t) & =\left[\sum_{a=0}^{\infty}\left(\frac{\left|v_{a}-t_{a}\right|}{a+1}\right)^{r_{a}}\right]^{\frac{1}{K}} \leq\left[\sum_{a=0}^{\infty}\left(\frac{\left|v_{a}-t_{a}^{b}\right|}{a+1}\right)^{r_{a}}\right]^{\frac{1}{K}}+\left[\sum_{a=0}^{\infty}\left(\frac{\left|t_{a}^{b}-t_{a}\right|}{a+1}\right)^{r_{a}}\right]^{\frac{1}{R}} \\
& \leq \operatorname{supinf}_{j \geq j} \psi\left(v-t^{b}\right) .
\end{aligned}
$$

Theorem 4.6 If $\left(r_{a}\right) \in \mathfrak{R}^{+\mathcal{N}} \cap \ell_{\infty}$ is increasing with $r_{0}>1$, then $\psi(v)=\sum_{a=0}^{\infty}\left(\frac{\left|\sum_{z=0}^{a} \Delta v_{z}\right|}{a+1}\right)^{r_{a}}$ does not satisfy the Fatou property for all $v \in \Xi(\Delta, r)$.

Proof Let the conditions be fulfilled and $\left\{t^{b}\right\} \subseteq(\Xi(\Delta, r))_{\psi}$ with $\lim _{b \rightarrow \infty} \psi\left(t^{b}-t\right)=0$. Since the space $(\Xi(\Delta, r))_{\psi}$ is a pre-quasi closed space, then $t \in(\Xi(\Delta, r))_{\psi}$. Then, for any $v \in$ $(\Xi(\Delta, r))_{\psi}$, we have

$$
\begin{aligned}
\psi(v-t) & =\sum_{a=0}^{\infty}\left(\frac{\left|v_{a}-t_{a}\right|}{a+1}\right)^{r_{a}} \leq 2^{\sup _{a} r_{a}-1}\left[\sum_{a=0}^{\infty}\left(\frac{\left|v_{a}-t_{a}^{b}\right|}{a+1}\right)^{r_{a}}+\sum_{a=0}^{\infty}\left(\frac{\left|t_{a}^{b}-t_{a}\right|}{a+1}\right)^{r_{a}}\right] \\
& \leq 2^{\sup _{a} r_{a}-1} \sup _{j} \inf _{b \geq j} \psi\left(v-t^{b}\right) .
\end{aligned}
$$

Hence, $\psi$ does not satisfy the Fatou property.

Theorem 4.7 If $\left(r_{a}\right) \in(0,1)^{\mathcal{N}}$ is increasing, then $\psi(v)=\left[\sum_{a=0}^{\infty}\left(\frac{\left|\sum_{z=0}^{a} \Delta v_{z}\right|}{a+1}\right)^{r_{a}}\right]^{\frac{1}{r_{0}}}$ does not satisfy the Fatou property for all $v \in \Xi(\Delta, r)$.

Proof Let the conditions be fulfilled and $\left\{t^{b}\right\} \subseteq(\Xi(\Delta, r))_{\psi}$ with $\lim _{b \rightarrow \infty} \psi\left(t^{b}-t\right)=0$. Since the space $(\Xi(\Delta, r))_{\psi}$ is a pre-quasi closed space, then $t \in(\Xi(\Delta, r))_{\psi}$. Then, for any $v \in$ $(\Xi(\Delta, r))_{\psi}$, we have

$$
\begin{aligned}
\psi(v-t) & =\left[\sum_{a=0}^{\infty}\left(\frac{\left|v_{a}-t_{a}\right|}{a+1}\right)^{r_{a}}\right]^{\frac{1}{r_{0}}} \\
& \leq 2^{\frac{1}{r_{0}}-1}\left(\left[\sum_{a=0}^{\infty}\left(\frac{\left|v_{a}-t_{a}^{b}\right|}{a+1}\right)^{r_{a}}\right]^{\frac{1}{r_{0}}}+\left[\sum_{a=0}^{\infty}\left(\frac{\left|t_{a}^{b}-t_{a}\right|}{a+1}\right)^{r_{a}}\right]^{\frac{1}{r_{0}}}\right) \\
& \leq 2^{\frac{1}{r_{0}}-1} \sup _{j} \inf _{b \geq j} \psi\left(v-t^{b}\right) .
\end{aligned}
$$

Hence, $\psi$ does not satisfy the Fatou property. 
Example 4.8 The function $\psi(v)=\left[\sum_{a=0}^{\infty}\left(\frac{\left|\sum_{z=0}^{a} \Delta v_{z}\right|}{a+1}\right)^{r_{a}}\right]^{\frac{1}{K}}$ is a pre-quasi norm, not quasi, and not a norm for each $v \in \Xi(\Delta, r)$.

Example 4.9 The function $\psi(v)=\left[\sum_{a=0}^{\infty}\left(\frac{\left|\sum_{z=0}^{a} \Delta v_{z}\right|}{a+1}\right)^{r}\right]^{\frac{1}{r}}$ is a pre-quasi norm, a quasi norm, and not a norm on $\Xi(\Delta,(r))$ for $0<r<1$.

Example 4.10 The function $\psi(v)=\inf \left\{\kappa>0: \sum_{a=0}^{\infty}\left(\frac{\left|\sum_{z=0}^{a} \Delta v_{z}\right|}{(a+1) \kappa}\right)^{r_{a}} \leq 1\right\}$ is a pre-quasi norm, a quasi norm, and a norm on $\Xi(\Delta, r)$.

\section{Fixed points of Kannan pre-quasi contraction mapping}

Now, we give the definition of Kannan $\psi$-Lipschitzian mapping in the pre-quasi normed (sss). We examine the sufficient conditions on $(\Xi(\Delta, r))_{\psi}$ equipped with definite pre-quasi norm such that there is a unique fixed point of the Kannan pre-quasi norm contraction mapping.

Definition 5.1 An operator $W: \mathfrak{A}_{\psi} \rightarrow \mathfrak{A}_{\psi}$ is called Kannan $\psi$-Lipschitzian if there is $\xi \geq 0$ such that

$$
\psi(W v-W t) \leq \xi(\psi(W v-v)+\psi(W t-t))
$$

for all $v, t \in \mathfrak{A}_{\psi}$. The operator $W$ is called:

(1) Kannan $\psi$-contraction if $\xi \in\left[0, \frac{1}{2}\right)$.

(2) Kannan $\psi$-nonexpansive if $\xi=\frac{1}{2}$.

A vector $v \in \mathfrak{A}_{\psi}$ is named a fixed point of $W$ when $W(v)=v$.

Theorem 5.2 If $\left(r_{a}\right) \in \mathfrak{R}^{+\mathcal{N}} \cap \ell_{\infty}$ is increasing and $W:(\Xi(\Delta, r))_{\psi} \rightarrow(\Xi(\Delta, r))_{\psi}$ is a Kannan $\psi$-contraction mapping, where $\psi(v)=\left[\sum_{a=0}^{\infty}\left(\frac{\left|\sum_{z=0}^{a} \Delta v_{z}\right|}{a+1}\right)^{r a}\right]^{\frac{1}{K}}$ for all $v \in \Xi(\Delta, r)$, hence W has a unique fixed point.

Proof Suppose that the conditions are satisfied. For any $v \in \Xi(\Delta, r)$, then $W^{p} v \in \Xi(\Delta, r)$. As $W$ is a Kannan $\psi$-contraction mapping, one has

$$
\begin{aligned}
& \psi\left(W^{p+1} v-W^{p} v\right) \leq \xi\left(\psi\left(W^{p+1} v-W^{p} v\right)+\psi\left(W^{p} v-W^{p-1} v\right)\right) \\
& \Rightarrow \psi\left(W^{p+1} v-W^{p} v\right) \leq \frac{\xi}{1-\xi} \psi\left(W^{p} v-W^{p-1} v\right) \\
& \leq\left(\frac{\xi}{1-\xi}\right)^{2} \psi\left(W^{p-1} v-W^{p-2} v\right) \\
& \leq \ldots \leq\left(\frac{\xi}{1-\xi}\right)^{p} \psi(W v-v) .
\end{aligned}
$$

Hence, for all $p, q \in \mathcal{N}$ with $q>p$, we get

$$
\begin{aligned}
\psi\left(W^{p} v-W^{q} v\right) & \leq \xi\left(\psi\left(W^{p} v-W^{p-1} v\right)+\psi\left(W^{q} v-W^{q-1} v\right)\right) \\
& \leq \xi\left(\left(\frac{\xi}{1-\xi}\right)^{p-1}+\left(\frac{\xi}{1-\xi}\right)^{q-1}\right) \psi(W v-v) .
\end{aligned}
$$


So, $\left\{W^{p} v\right\}$ is a Cauchy sequence in $(\Xi(\Delta, r))_{\psi}$. As the space $(\Xi(\Delta, r))_{\psi}$ is a pre-quasi Banach space, there is $t \in(\Xi(\Delta, r))_{\psi}$ such that $\lim _{p \rightarrow \infty} W^{p} v=t$. To prove that $W t=t$, since $\psi$ has the Fatou property, one can see

$$
\psi(W t-t) \leq \sup _{i} \inf _{p \geq i} \psi\left(W^{p+1} v-W^{p} v\right) \leq \operatorname{supinf}_{i}\left(\frac{\xi}{1-\xi}\right)^{p} \psi(W v-v)=0,
$$

hence $W t=t$. Then $t$ is a fixed point of $W$. To show that the fixed point is unique, let us have two different fixed points $b, t \in(\Xi(\Delta, r))_{\psi}$ of $W$. Hence, we obtain

$$
\psi(b-t) \leq \psi(W b-W t) \leq \xi(\psi(W b-b)+\psi(W t-t))=0 .
$$

Therefore, $b=t$.

Corollary 5.3 Let $\left(r_{a}\right) \in \mathfrak{R}^{+\mathcal{N}} \cap \ell_{\infty}$ be increasing and $W:(\Xi(\Delta, r))_{\psi} \rightarrow(\Xi(\Delta, r))_{\psi}$ be a Kannan $\psi$-contraction mapping, where $\psi(v)=\left[\sum_{a=0}^{\infty}\left(\frac{\left|\sum_{z=0}^{a} \Delta v_{z}\right|}{a+1}\right)^{r_{a}}\right]^{\frac{1}{K}}$ for all $v \in \Xi(\Delta, r)$, then $W$ has one and only one fixed point $b$ with $\psi\left(W^{p} v-b\right) \leq \xi\left(\frac{\xi}{1-\xi}\right)^{p-1} \psi(W v-v)$.

Proof Let the conditions be satisfied. By Theorem 5.2, there is a unique fixed point $b$ of $W$. Consequently, we have

$$
\begin{aligned}
\psi\left(W^{p} v-b\right) & =\psi\left(W^{p} v-W b\right) \leq \xi\left(\psi\left(W^{p} v-W^{p-1} v\right)+\psi(W b-b)\right) \\
& =\xi\left(\frac{\xi}{1-\xi}\right)^{p-1} \psi(W v-v) .
\end{aligned}
$$

Definition 5.4 Assume that $\mathfrak{A}_{\psi}$ is a pre-quasi normed (sss) and $W: \mathfrak{A}_{\psi} \rightarrow \mathfrak{A}_{\psi}$. The operator $W$ is called $\psi$-sequentially continuous at $b \in \mathfrak{A}_{\psi}$ if and only if, when $\lim _{a \rightarrow \infty} \psi\left(v_{a}-\right.$ $b)=0$, then $\lim _{a \rightarrow \infty} \psi\left(W v_{a}-W b\right)=0$.

Theorem 5.5 Let $\left(r_{a}\right) \in \mathfrak{R}^{+\mathcal{N}} \cap \ell_{\infty}$ be increasing with $r_{0}>1$, and let $W:(\Xi(\Delta, r))_{\psi} \rightarrow$ $(\Xi(\Delta, r))_{\psi}$, where $\psi(v)=\sum_{a=0}^{\infty}\left(\frac{\left|\sum_{z=0}^{a} \Delta v_{z}\right|}{a+1}\right)^{r}$ for all $v \in \Xi(\Delta, r)$. The point $g \in(\Xi(\Delta, r))_{\psi}$ is the only fixed point of $W$ if the following conditions are satisfied:

(a) $W$ is a Kannan $\psi$-contraction mapping;

(b) $W$ is $\psi$-sequentially continuous at a point $g \in(\Xi(\Delta, r))_{\psi}$;

(c) There is $v \in(\Xi(\Delta, r))_{\psi}$ so that the sequence of iterates $\left\{W^{p} v\right\}$ has a subsequence $\left\{W^{p_{i}} v\right\}$ converging to $g$.

Proof Let the conditions be verified. If $g$ is not a fixed point of $W$, then $W g \neq g$. By conditions (b) and (c), we have

$$
\lim _{p_{i} \rightarrow \infty} \psi\left(W^{p_{i}} v-g\right)=0 \text { and } \lim _{p_{i} \rightarrow \infty} \psi\left(W^{p_{i}+1} v-W g\right)=0 .
$$

As the operator $W$ is a Kannan $\psi$-contraction, one can see

$$
\begin{aligned}
0<\psi(W g-g)= & \psi\left(\left(W g-W^{p_{i}+1} v\right)+\left(W^{p_{i}} v-g\right)+\left(W^{p_{i}+1} v-W^{p_{i}} v\right)\right) \\
\leq & 2^{2 \sup _{i} r_{i}-2} \psi\left(W^{p_{i}+1} v-W g\right)+2^{2 \sup _{i} r_{i}-2} \psi\left(W^{p_{i}} v-g\right) \\
& +2^{\sup _{i} r_{i}-1} \xi\left(\frac{\xi}{1-\xi}\right)^{p_{i}-1} \psi(W v-v) .
\end{aligned}
$$


As $p_{i} \rightarrow \infty$, we have a contradiction. Therefore, $g$ is a fixed point of $W$. To explain that the fixed point $g$ is unique, let us have two different fixed points $g, b \in(\Xi(\Delta, r))_{\psi}$ of $W$. Hence, we obtain

$$
\psi(g-b) \leq \psi(W g-W b) \leq \xi(\psi(W g-g)+\psi(W b-b))=0 .
$$

Therefore, $g=b$.

Theorem 5.6 Let $\left(r_{a}\right) \in(0,1)^{\mathcal{N}}$ be increasing, and let $W:(\Xi(\Delta, r))_{\psi} \rightarrow(\Xi(\Delta, r))_{\psi}$, where $\psi(v)=\left[\sum_{a=0}^{\infty}\left(\frac{\left|\sum_{z=0}^{a} \Delta v_{z}\right|}{a+1}\right)^{r_{a}}\right]^{\frac{1}{r_{0}}}$ for all $v \in \Xi(\Delta, r)$. The point $g \in(\Xi(\Delta, r))_{\psi}$ is the only fixed point of $W$ if the following conditions are satisfied:

(a) $W$ is a Kannan $\psi$-contraction mapping;

(b) $W$ is $\psi$-sequentially continuous at $g \in(\Xi(\Delta, r))_{\psi}$;

(c) There is $v \in(\Xi(\Delta, r))_{\psi}$ such that the sequence of iterates $\left\{W^{p} v\right\}$ has a subsequence $\left\{W^{p_{i}} v\right\}$ converging to $g$.

Proof Let the conditions be verified. If $g$ is not a fixed point of $W$, then $W g \neq g$. By conditions (b) and (c), we have

$$
\lim _{p_{i} \rightarrow \infty} \psi\left(W^{p_{i}} v-g\right)=0 \text { and } \lim _{p_{i} \rightarrow \infty} \psi\left(W^{p_{i}+1} v-W g\right)=0
$$

As the operator $W$ is a Kannan $\psi$-contraction, one can see

$$
\begin{aligned}
0<\psi(W g-g)= & \psi\left(\left(W g-W^{p_{i}+1} v\right)+\left(W^{p_{i}} v-g\right)+\left(W^{p_{i}+1} v-W^{p_{i}} v\right)\right) \\
\leq & 2^{2 r_{0}^{-1}-2} \psi\left(W^{p_{i}+1} v-W g\right)+2^{2 r_{0}^{-1}-2} \psi\left(W^{p_{i}} v-g\right) \\
& +2^{r_{0}^{-1}-1} \xi\left(\frac{\xi}{1-\xi}\right)^{p_{i}-1} \psi(W v-v) .
\end{aligned}
$$

As $p_{i} \rightarrow \infty$, we have a contradiction. Therefore, $g$ is a fixed point of $W$. To explain that the fixed point $g$ is unique, let us have two different fixed points $g, b \in(\Xi(\Delta, r))_{\psi}$ of $W$. Hence, we obtain

$$
\psi(g-b) \leq \psi(W g-W b) \leq \xi(\psi(W g-g)+\psi(W b-b))=0
$$

Therefore, $g=b$.

Example 5.7 Let $W:\left(\Xi\left(\Delta,\left(\frac{a+1}{2 a+4}\right)_{a=0}^{\infty}\right)\right)_{\psi} \rightarrow\left(\Xi\left(\Delta,\left(\frac{a+1}{2 a+4}\right)_{a=0}^{\infty}\right)\right)_{\psi}$, where $\psi(v)=$ $\sum_{a \in \mathcal{N}}\left|\frac{v_{a}}{a+1}\right| \frac{a+1}{2 a+4}$ for all $v \in \Xi\left(\Delta,\left(\frac{a+1}{2 a+4}\right)_{a=0}^{\infty}\right)$ and

$$
W(v)= \begin{cases}\frac{v}{18}, & \psi(v) \in[0,1), \\ \frac{v}{20}, & \psi(v) \in[1, \infty) .\end{cases}
$$

Since for all $v_{1}, v_{2} \in\left(\Xi\left(\Delta,\left(\frac{a+1}{2 a+4}\right)_{a=0}^{\infty}\right)\right)_{\psi}$ with $\psi\left(v_{1}\right), \psi\left(v_{2}\right) \in[0,1)$, we have

$$
\psi\left(W v_{1}-W v_{2}\right)=\psi\left(\frac{v_{1}}{18}-\frac{v_{2}}{18}\right) \leq \frac{1}{\sqrt[4]{17}}\left(\psi\left(\frac{17 v_{1}}{18}\right)+\psi\left(\frac{17 v_{2}}{18}\right)\right)
$$




$$
=\frac{1}{\sqrt[4]{17}}\left(\psi\left(W v_{1}-v_{1}\right)+\psi\left(W v_{2}-v_{2}\right)\right)
$$

For all $v_{1}, v_{2} \in\left(\Xi\left(\Delta,\left(\frac{a+1}{2 a+4}\right)_{a=0}^{\infty}\right)\right)_{\psi}$ with $\psi\left(v_{1}\right), \psi\left(v_{2}\right) \in[1, \infty)$, we have

$$
\begin{aligned}
\psi\left(W v_{1}-W v_{2}\right) & =\psi\left(\frac{v_{1}}{20}-\frac{v_{2}}{20}\right) \leq \frac{1}{\sqrt[4]{19}}\left(\psi\left(\frac{19 v_{1}}{20}\right)+\psi\left(\frac{19 v_{2}}{20}\right)\right) \\
& =\frac{1}{\sqrt[4]{19}}\left(\psi\left(W v_{1}-v_{1}\right)+\psi\left(W v_{2}-v_{2}\right)\right)
\end{aligned}
$$

For all $v_{1}, v_{2} \in\left(\Xi\left(\Delta,\left(\frac{a+1}{2 a+4}\right)_{a=0}^{\infty}\right)\right)_{\psi}$ with $\psi\left(v_{1}\right) \in[0,1)$ and $\psi\left(v_{2}\right) \in[1, \infty)$, we have

$$
\begin{aligned}
\psi\left(W v_{1}-W v_{2}\right) & =\psi\left(\frac{v_{1}}{18}-\frac{v_{2}}{20}\right) \leq \frac{1}{\sqrt[4]{17}} \psi\left(\frac{17 v_{1}}{18}\right)+\frac{1}{\sqrt[4]{19}} \psi\left(\frac{19 v_{2}}{20}\right) \\
& \leq \frac{1}{\sqrt[4]{17}}\left(\psi\left(\frac{17 v_{1}}{18}\right)+\psi\left(\frac{19 v_{2}}{20}\right)\right) \\
& =\frac{1}{\sqrt[4]{17}}\left(\psi\left(W v_{1}-v_{1}\right)+\psi\left(W v_{2}-v_{2}\right)\right) .
\end{aligned}
$$

Therefore, the map $W$ is a Kannan $\psi$-contraction mapping. Since $\psi$ satisfies the Fatou property, by Theorem 5.2, the map $W$ has a unique fixed point $\theta \in\left(\Xi\left(\Delta,\left(\frac{a+1}{2 a+4}\right)_{a=0}^{\infty}\right)\right)_{\psi}$.

Let $\left\{v^{(n)}\right\} \subseteq\left(\Xi\left(\Delta,\left(\frac{a+1}{2 a+4}\right)_{a=0}^{\infty}\right)\right)_{\psi}$ be such that $\lim _{n \rightarrow \infty} \psi\left(v^{(n)}-v^{(0)}\right)=0$, where $v^{(0)} \in$ $\left(\Xi\left(\Delta,\left(\frac{a+1}{2 a+4}\right)_{a=0}^{\infty}\right)\right)_{\psi}$ with $\psi\left(v^{(0)}\right)=1$. Since the pre-quasi norm $\psi$ is continuous, we have

$$
\lim _{n \rightarrow \infty} \psi\left(W v^{(n)}-W \nu^{(0)}\right)=\lim _{n \rightarrow \infty} \psi\left(\frac{\nu^{(n)}}{18}-\frac{\nu^{(0)}}{20}\right)=\psi\left(\frac{\nu^{(0)}}{180}\right)>0
$$

Hence $W$ is not $\psi$-sequentially continuous at $v^{(0)}$. So, the map $W$ is not continuous at $v^{(0)}$.

If $\psi(v)=\left[\sum_{a \in \mathcal{N}}\left|\frac{v_{a}}{a+1}\right| \frac{a+1}{2 a+4}\right]^{4}$ for all $v \in \Xi\left(\Delta,\left(\frac{a+1}{2 a+4}\right)_{a=0}^{\infty}\right)$. Since for all $v_{1}, v_{2} \in(\Xi(\Delta$, $\left.\left.\left(\frac{a+1}{2 a+4}\right)_{a=0}^{\infty}\right)\right)_{\psi}$ with $\psi\left(v_{1}\right), \psi\left(v_{2}\right) \in[0,1)$, we have

$$
\begin{aligned}
\psi\left(W v_{1}-W v_{2}\right) & =\psi\left(\frac{v_{1}}{18}-\frac{v_{2}}{18}\right) \leq \frac{8}{17}\left(\psi\left(\frac{17 v_{1}}{18}\right)+\psi\left(\frac{17 v_{2}}{18}\right)\right) \\
& =\frac{8}{17}\left(\psi\left(W v_{1}-v_{1}\right)+\psi\left(W v_{2}-v_{2}\right)\right) .
\end{aligned}
$$

For all $v_{1}, v_{2} \in\left(\Xi\left(\Delta,\left(\frac{a+1}{2 a+4}\right)_{a=0}^{\infty}\right)\right)_{\psi}$ with $\psi\left(v_{1}\right), \psi\left(v_{2}\right) \in[1, \infty)$, we have

$$
\begin{aligned}
\psi\left(W v_{1}-W v_{2}\right) & =\psi\left(\frac{v_{1}}{20}-\frac{v_{2}}{20}\right) \leq \frac{8}{19}\left(\psi\left(\frac{19 v_{1}}{20}\right)+\psi\left(\frac{19 v_{2}}{20}\right)\right) \\
& =\frac{8}{19}\left(\psi\left(W v_{1}-v_{1}\right)+\psi\left(W v_{2}-v_{2}\right)\right) .
\end{aligned}
$$

For all $v_{1}, v_{2} \in\left(\Xi\left(\Delta,\left(\frac{a+1}{2 a+4}\right)_{a=0}^{\infty}\right)\right)_{\psi}$ with $\psi\left(v_{1}\right) \in[0,1)$ and $\psi\left(v_{2}\right) \in[1, \infty)$, we have

$$
\begin{aligned}
\psi\left(W v_{1}-W v_{2}\right) & =\psi\left(\frac{v_{1}}{18}-\frac{v_{2}}{20}\right) \leq \frac{8}{17} \psi\left(\frac{17 v_{1}}{18}\right)+\frac{8}{19} \psi\left(\frac{19 v_{2}}{20}\right) \\
& \leq \frac{8}{17}\left(\psi\left(\frac{17 v_{1}}{18}\right)+\psi\left(\frac{19 v_{2}}{20}\right)\right)
\end{aligned}
$$




$$
=\frac{8}{17}\left(\psi\left(W v_{1}-v_{1}\right)+\psi\left(W v_{2}-v_{2}\right)\right) .
$$

Therefore, the map $W$ is a Kannan $\psi$-contraction mapping and

$$
W^{p}(v)= \begin{cases}\frac{v}{18^{p}}, & \psi(v) \in[0,1), \\ \frac{v}{20^{p}}, & \psi(v) \in[1, \infty) .\end{cases}
$$

It is clear that $W$ is $\psi$-sequentially continuous at $\theta \in\left(\Xi\left(\Delta,\left(\frac{a+1}{2 a+4}\right)_{a=0}^{\infty}\right)\right)_{\psi}$ and $\left\{W^{p} v\right\}$ has a subsequence $\left\{W^{p_{i}} v\right\}$ converging to $\theta$. By Theorem 5.5 , the point $\theta \in\left(\Xi\left(\Delta,\left(\frac{a+1}{2 a+4}\right)_{a=0}^{\infty}\right)\right)_{\psi}$ is the only fixed point of $W$.

Example 5.8 Let $W:\left(\Xi\left(\Delta,\left(\frac{2 a+3}{a+2}\right)_{a=0}^{\infty}\right)\right)_{\psi} \rightarrow\left(\Xi\left(\Delta,\left(\frac{2 a+3}{a+2}\right)_{a=0}^{\infty}\right)\right)_{\psi}$, where $\psi(v)=$ $\sqrt{\sum_{a \in \mathcal{N}}\left|\frac{v_{a}}{a+1}\right| \frac{2 a+3}{a+2}}$ for all $v \in \Xi\left(\Delta,\left(\frac{2 a+3}{a+2}\right)_{a=0}^{\infty}\right)$ and

$$
W(v)= \begin{cases}\frac{v}{4}, & \psi(v) \in[0,1), \\ \frac{v}{5}, & \psi(v) \in[1, \infty) .\end{cases}
$$

Since for all $v_{1}, v_{2} \in\left(\Xi\left(\Delta,\left(\frac{2 a+3}{a+2}\right)_{a=0}^{\infty}\right)\right)_{\psi}$ with $\psi\left(v_{1}\right), \psi\left(v_{2}\right) \in[0,1)$, we have

$$
\begin{aligned}
\psi\left(W v_{1}-W v_{2}\right) & =\psi\left(\frac{v_{1}}{4}-\frac{v_{2}}{4}\right) \leq \frac{1}{\sqrt[4]{27}}\left(\psi\left(\frac{3 v_{1}}{4}\right)+\psi\left(\frac{3 v_{2}}{4}\right)\right) \\
& =\frac{1}{\sqrt[4]{27}}\left(\psi\left(W v_{1}-v_{1}\right)+\psi\left(W v_{2}-v_{2}\right)\right)
\end{aligned}
$$

For all $v_{1}, v_{2} \in\left(\Xi\left(\Delta,\left(\frac{2 a+3}{a+2}\right)_{a=0}^{\infty}\right)\right)_{\psi}$ with $\psi\left(v_{1}\right), \psi\left(v_{2}\right) \in[1, \infty)$, we have

$$
\begin{aligned}
\psi\left(W v_{1}-W v_{2}\right) & =\psi\left(\frac{v_{1}}{5}-\frac{v_{2}}{5}\right) \leq \frac{1}{\sqrt[4]{64}}\left(\psi\left(\frac{4 v_{1}}{5}\right)+\psi\left(\frac{4 v_{2}}{5}\right)\right) \\
& =\frac{1}{\sqrt[4]{64}}\left(\psi\left(W v_{1}-v_{1}\right)+\psi\left(W v_{2}-v_{2}\right)\right)
\end{aligned}
$$

For all $v_{1}, v_{2} \in\left(\Xi\left(\Delta,\left(\frac{2 a+3}{a+2}\right)_{a=0}^{\infty}\right)\right)_{\psi}$ with $\psi\left(v_{1}\right) \in[0,1)$ and $\psi\left(v_{2}\right) \in[1, \infty)$, we have

$$
\begin{aligned}
\psi\left(W v_{1}-W v_{2}\right) & =\psi\left(\frac{v_{1}}{4}-\frac{v_{2}}{5}\right) \leq \frac{1}{\sqrt[4]{27}} \psi\left(\frac{3 v_{1}}{4}\right)+\frac{1}{\sqrt[4]{64}} \psi\left(\frac{4 v_{2}}{5}\right) \\
& \leq \frac{1}{\sqrt[4]{27}}\left(\psi\left(\frac{3 v_{1}}{4}\right)+\psi\left(\frac{4 v_{2}}{5}\right)\right) \\
& =\frac{1}{\sqrt[4]{27}}\left(\psi\left(W v_{1}-v_{1}\right)+\psi\left(W v_{2}-v_{2}\right)\right) .
\end{aligned}
$$

Therefore, the map $W$ is a Kannan $\psi$-contraction mapping. Since $\psi$ satisfies the Fatou property, by Theorem 5.2, the map $W$ has a unique fixed point $\theta \in\left(\Xi\left(\Delta,\left(\frac{2 a+3}{a+2}\right)_{a=0}^{\infty}\right)\right)_{\psi}$.

Let $\left\{v^{(n)}\right\} \subseteq\left(\Xi\left(\Delta,\left(\frac{2 a+3}{a+2}\right)_{a=0}^{\infty}\right)\right)_{\psi}$ be such that $\lim _{n \rightarrow \infty} \psi\left(v^{(n)}-v^{(0)}\right)=0$, where $v^{(0)} \in$ $\left(\Xi\left(\Delta,\left(\frac{2 a+3}{a+2}\right)_{a=0}^{\infty}\right)\right)_{\psi}$ with $\psi\left(v^{(0)}\right)=1$. Since the pre-quasi norm $\psi$ is continuous, we have

$$
\lim _{n \rightarrow \infty} \psi\left(W v^{(n)}-W v^{(0)}\right)=\lim _{n \rightarrow \infty} \psi\left(\frac{\nu^{(n)}}{4}-\frac{\nu^{(0)}}{5}\right)=\psi\left(\frac{\nu^{(0)}}{20}\right)>0 .
$$


Hence $W$ is not $\psi$-sequentially continuous at $v^{(0)}$. So, the map $W$ is not continuous at $v^{(0)}$.

If $\psi(v)=\sum_{a \in \mathcal{N}}\left|\frac{v_{a}}{a+1}\right|^{\frac{2 a+3}{a+2}}$ for all $v \in \Xi\left(\Delta,\left(\frac{2 a+3}{a+2}\right)_{a=0}^{\infty}\right)$. Since for all $v_{1}, v_{2} \in\left(\Xi\left(\Delta,\left(\frac{2 a+3}{a+2}\right)_{a=0}^{\infty}\right)\right)_{\psi}$ with $\psi\left(v_{1}\right), \psi\left(v_{2}\right) \in[0,1)$, we have

$$
\begin{aligned}
\psi\left(W v_{1}-W v_{2}\right) & =\psi\left(\frac{v_{1}}{4}-\frac{v_{2}}{4}\right) \leq \frac{2}{\sqrt{27}}\left(\psi\left(\frac{3 v_{1}}{4}\right)+\psi\left(\frac{3 v_{2}}{4}\right)\right) \\
& =\frac{2}{\sqrt{27}}\left(\psi\left(W v_{1}-v_{1}\right)+\psi\left(W v_{2}-v_{2}\right)\right) .
\end{aligned}
$$

For all $v_{1}, v_{2} \in\left(\Xi\left(\Delta,\left(\frac{2 a+3}{a+2}\right)_{a=0}^{\infty}\right)\right)_{\psi}$ with $\psi\left(v_{1}\right), \psi\left(v_{2}\right) \in[1, \infty)$, we have

$$
\begin{aligned}
\psi\left(W v_{1}-W v_{2}\right) & =\psi\left(\frac{v_{1}}{5}-\frac{v_{2}}{5}\right) \leq \frac{1}{4}\left(\psi\left(\frac{4 v_{1}}{5}\right)+\psi\left(\frac{4 v_{2}}{5}\right)\right) \\
& =\frac{1}{4}\left(\psi\left(W v_{1}-v_{1}\right)+\psi\left(W v_{2}-v_{2}\right)\right) .
\end{aligned}
$$

For all $v_{1}, v_{2} \in\left(\Xi\left(\Delta,\left(\frac{2 a+3}{a+2}\right)_{a=0}^{\infty}\right)\right)_{\psi}$ with $\psi\left(v_{1}\right) \in[0,1)$ and $\psi\left(v_{2}\right) \in[1, \infty)$, we have

$$
\begin{aligned}
\psi\left(W \nu_{1}-W v_{2}\right) & =\psi\left(\frac{v_{1}}{4}-\frac{v_{2}}{5}\right) \leq \frac{2}{\sqrt{27}} \psi\left(\frac{3 v_{1}}{4}\right)+\frac{1}{4} \psi\left(\frac{4 v_{2}}{5}\right) \\
& \leq \frac{2}{\sqrt{27}}\left(\psi\left(\frac{3 v_{1}}{4}\right)+\psi\left(\frac{4 v_{2}}{5}\right)\right) \\
& =\frac{2}{\sqrt{27}}\left(\psi\left(W v_{1}-v_{1}\right)+\psi\left(W v_{2}-v_{2}\right)\right) .
\end{aligned}
$$

Therefore, the map $W$ is a Kannan $\psi$-contraction mapping and

$$
W^{p}(v)= \begin{cases}\frac{v}{4^{p}}, & \psi(v) \in[0,1) \\ \frac{v}{5^{p}}, & \psi(v) \in[1, \infty)\end{cases}
$$

It is clear that $W$ is $\psi$-sequentially continuous at $\theta \in\left(\Xi\left(\Delta,\left(\frac{2 a+3}{a+2}\right)_{a=0}^{\infty}\right)\right)_{\psi}$ and $\left\{W^{p} v\right\}$ has a subsequence $\left\{W^{p_{i}} v\right\}$ converging to $\theta$. By Theorem 5.5 , the point $\theta \in\left(\Xi\left(\Delta,\left(\frac{2 a+3}{a+2}\right)_{a=0}^{\infty}\right)\right)_{\psi}$ is the only fixed point of $W$.

Example 5.9 Let $W:\left(\Xi\left(\Delta,\left(\frac{a+1}{2 a+4}\right)_{a=0}^{\infty}\right)\right)_{\psi} \rightarrow\left(\Xi\left(\Delta,\left(\frac{a+1}{2 a+4}\right)_{a=0}^{\infty}\right)\right)_{\psi}$, where $\psi(v)=$ $\left[\sum_{a \in \mathcal{N}}\left|\frac{v_{a}}{a+1}\right| \frac{a+1}{2 a+4}\right]^{4}$ for all $v \in \Xi\left(\Delta,\left(\frac{a+1}{2 a+4}\right)_{a=0}^{\infty}\right)$ and

$$
W(v)= \begin{cases}\frac{1}{18}\left(e_{0}+v\right), & v_{0} \in\left(-\infty, \frac{1}{17}\right), \\ \frac{1}{17} e_{0}, & v_{0}=\frac{1}{17}, \\ \frac{1}{18} e_{0}, & v_{0} \in\left(\frac{1}{17}, \infty\right)\end{cases}
$$

Since for all $v, t \in\left(\Xi\left(\Delta,\left(\frac{a+1}{2 a+4}\right)_{a=0}^{\infty}\right)\right)_{\psi}$ with $v_{0}, t_{0} \in\left(-\infty, \frac{1}{17}\right)$, we have

$$
\psi(W v-W t)=\psi\left(\frac{1}{18}\left(v_{0}-t_{0}, v_{1}-t_{1}, v_{2}-t_{2}, \ldots\right)\right) \leq \frac{8}{17}\left(\psi\left(\frac{17 v}{18}\right)+\psi\left(\frac{17 t}{18}\right)\right)
$$




$$
\leq \frac{8}{17}(\psi(W v-v)+\psi(W t-t))
$$

For all $v, t \in\left(\Xi\left(\Delta,\left(\frac{a+1}{2 a+4}\right)_{a=0}^{\infty}\right)\right)_{\psi}$ with $v_{0}, t_{0} \in\left(\frac{1}{17}, \infty\right)$, then for any $\varepsilon>0$ we have

$$
\psi(W v-W t)=0 \leq \varepsilon(\psi(W v-v)+\psi(W t-t))
$$

For all $v, t \in\left(\Xi\left(\Delta,\left(\frac{a+1}{2 a+4}\right)_{a=0}^{\infty}\right)\right)_{\psi}$ with $v_{0} \in\left(-\infty, \frac{1}{17}\right)$ and $t_{0} \in\left(\frac{1}{17}, \infty\right)$, we have

$$
\begin{aligned}
\psi(W v-W t) & =\psi\left(\frac{v}{18}\right) \leq \frac{1}{17} \psi\left(\frac{17 v}{18}\right)=\frac{1}{17} \psi(W v-v) \\
& \leq \frac{1}{17}(\psi(W v-v)+\psi(W t-t)) .
\end{aligned}
$$

Therefore, the map $W$ is a Kannan $\psi$-contraction mapping. It is clear that $W$ is $\psi$ sequentially continuous at $\frac{1}{17} e_{0} \in\left(\Xi\left(\Delta,\left(\frac{a+1}{2 a+4}\right)_{a=0}^{\infty}\right)\right)_{\psi}$, and there is $v \in\left(\Xi\left(\Delta,\left(\frac{a+1}{2 a+4}\right)_{a=0}^{\infty}\right)\right)_{\psi}$ with $v_{0} \in\left(-\infty, \frac{1}{17}\right)$ such that the sequence of iterates $\left\{W^{p} v\right\}=\left\{\sum_{n=1}^{p} \frac{1}{18^{n}} e_{0}+\frac{1}{18^{p}} v\right\}$ has a subsequence $\left\{W^{p_{i}} \nu\right\}=\left\{\sum_{n=1}^{p_{i}} \frac{1}{18^{n}} e_{0}+\frac{1}{18^{p_{i}}} \nu\right\}$ converging to $\frac{1}{17} e_{0}$. By Theorem 5.5, the map $W$ has one fixed point $\frac{1}{17} e_{0} \in\left(\Xi\left(\Delta,\left(\frac{a+1}{2 a+4}\right)_{a=0}^{\infty}\right)\right)_{\psi}$. Note that $W$ is not continuous at $\frac{1}{17} e_{0} \in\left(\Xi\left(\Delta,\left(\frac{a+1}{2 a+4}\right)_{a=0}^{\infty}\right)\right)_{\psi}$.

If $\psi(v)=\sum_{a \in \mathcal{N}}\left|\frac{v_{a}}{a+1}\right| \frac{a+1}{2 a+4}$ for all $v \in \Xi\left(\Delta,\left(\frac{a+1}{2 a+4}\right)_{a=0}^{\infty}\right)$. Since for all $v, t \in\left(\Xi\left(\Delta,\left(\frac{a+1}{2 a+4}\right)_{a=0}^{\infty}\right)\right)_{\psi}$ with $v_{0}, t_{0} \in\left(-\infty, \frac{1}{17}\right)$, we have

$$
\begin{aligned}
\psi(W v-W t) & =\psi\left(\frac{1}{18}\left(v_{0}-t_{0}, v_{1}-t_{1}, v_{2}-t_{2}, \ldots\right)\right) \leq \frac{1}{\sqrt[4]{17}}\left(\psi\left(\frac{17 v}{18}\right)+\psi\left(\frac{17 t}{18}\right)\right) \\
& \leq \frac{1}{\sqrt[4]{17}}(\psi(W v-v)+\psi(W t-t))
\end{aligned}
$$

For all $v, t \in\left(\Xi\left(\Delta,\left(\frac{a+1}{2 a+4}\right)_{a=0}^{\infty}\right)\right)_{\psi}$ with $v_{0}, t_{0} \in\left(\frac{1}{17}, \infty\right)$, then for any $\varepsilon>0$ we have

$$
\psi(W v-W t)=0 \leq \varepsilon(\psi(W v-v)+\psi(W t-t)) .
$$

For all $v, t \in\left(\Xi\left(\Delta,\left(\frac{a+1}{2 a+4}\right)_{a=0}^{\infty}\right)\right)_{\psi}$ with $v_{0} \in\left(-\infty, \frac{1}{17}\right)$ and $t_{0} \in\left(\frac{1}{17}, \infty\right)$, we have

$$
\begin{aligned}
\psi(W v-W t) & =\psi\left(\frac{v}{18}\right) \leq \frac{1}{\sqrt[4]{17}} \psi\left(\frac{17 v}{18}\right)=\frac{1}{\sqrt[4]{17}} \psi(W v-v) \\
& \leq \frac{1}{\sqrt[4]{17}}(\psi(W v-v)+\psi(W t-t))
\end{aligned}
$$

Therefore, the map $W$ is a Kannan $\psi$-contraction mapping. Since $\psi$ satisfies the Fatou property, by Theorem 5.2, the map $W$ has a unique fixed point $\frac{1}{17} e_{0} \in\left(\Xi\left(\Delta,\left(\frac{a+1}{2 a+4}\right)_{a=0}^{\infty}\right)\right)_{\psi}$.

Example 5.10 Let $W:\left(\Xi\left(\Delta,\left(\frac{2 a+3}{a+2}\right)_{a=0}^{\infty}\right)\right)_{\psi} \rightarrow\left(\Xi\left(\Delta,\left(\frac{2 a+3}{a+2}\right)_{a=0}^{\infty}\right)\right)_{\psi}$, where $\psi(v)=$ $\sum_{a \in \mathcal{N}}\left|\frac{v_{a}}{a+1}\right|^{2 a+2}$ for all $v \in \Xi\left(\Delta,\left(\frac{2 a+3}{a+2}\right)_{a=0}^{\infty}\right)$ and

$$
W(v)= \begin{cases}\frac{1}{4}\left(e_{1}+v\right), & v_{0} \in\left(-\infty, \frac{1}{3}\right), \\ \frac{1}{3} e_{1}, & v_{0}=\frac{1}{3}, \\ \frac{1}{4} e_{1}, & v_{0} \in\left(\frac{1}{3}, \infty\right) .\end{cases}
$$


Since for all $v, t \in\left(\Xi\left(\Delta,\left(\frac{2 a+3}{a+2}\right)_{a=0}^{\infty}\right)\right)_{\psi}$ with $v_{0}, t_{0} \in\left(-\infty, \frac{1}{3}\right)$, we have

$$
\begin{aligned}
\psi(W v-W t) & =\psi\left(\frac{1}{4}\left(v_{0}-t_{0}, v_{1}-t_{1}, v_{2}-t_{2}, \ldots\right)\right) \leq \frac{2}{\sqrt{27}}\left(\psi\left(\frac{3 v}{4}\right)+\psi\left(\frac{3 t}{4}\right)\right) \\
& \leq \frac{2}{\sqrt{27}}(\psi(W v-v)+\psi(W t-t)) .
\end{aligned}
$$

For all $v, t \in\left(\Xi\left(\Delta,\left(\frac{2 a+3}{a+2}\right)_{a=0}^{\infty}\right)\right)_{\psi}$ with $v_{0}, t_{0} \in\left(\frac{1}{3}, \infty\right)$, then for any $\varepsilon>0$ we have

$$
\psi(W v-W t)=0 \leq \varepsilon(\psi(W v-v)+\psi(W t-t)) .
$$

For all $v, t \in\left(\Xi\left(\Delta,\left(\frac{2 a+3}{a+2}\right)_{a=0}^{\infty}\right)\right)_{\psi}$ with $v_{0} \in\left(-\infty, \frac{1}{3}\right)$ and $t_{0} \in\left(\frac{1}{3}, \infty\right)$, we have

$$
\begin{aligned}
\psi(W v-W t) & =\psi\left(\frac{v}{4}\right) \leq \frac{1}{\sqrt{27}} \psi\left(\frac{3 v}{4}\right)=\frac{1}{\sqrt{27}} \psi(W v-v) \\
& \leq \frac{1}{\sqrt{27}}(\psi(W v-v)+\psi(W t-t)) .
\end{aligned}
$$

Therefore, the map $W$ is a Kannan $\psi$-contraction mapping. It is clear that $W$ is $\psi$ sequentially continuous at $\frac{1}{3} e_{1} \in\left(\Xi\left(\Delta,\left(\frac{2 a+3}{a+2}\right)_{a=0}^{\infty}\right)\right)_{\psi}$, and there is $v \in\left(\Xi\left(\Delta,\left(\frac{2 a+3}{a+2}\right)_{a=0}^{\infty}\right)\right)_{\psi}$ with $v_{0} \in\left(-\infty, \frac{1}{3}\right)$ such that the sequence of iterates $\left\{W^{p} v\right\}=\left\{\sum_{n=1}^{p} \frac{1}{4^{n}} e_{1}+\frac{1}{4^{p}} v\right\}$ has a subsequence $\left\{W^{p_{i}} v\right\}=\left\{\sum_{n=1}^{p_{i}} \frac{1}{4^{n}} e_{1}+\frac{1}{4^{p_{i}}} v\right\}$ converging to $\frac{1}{3} e_{1}$. By Theorem 5.5 , the map $W$ has one fixed point $\frac{1}{3} e_{1} \in\left(\Xi\left(\Delta,\left(\frac{2 a+3}{a+2}\right)_{a=0}^{\infty}\right)\right)_{\psi}$. Note that $W$ is not continuous at $\frac{1}{3} e_{1} \in$ $\left(\Xi\left(\Delta,\left(\frac{2 a+3}{a+2}\right)_{a=0}^{\infty}\right)\right)_{\psi}$.

If $\psi(v)=\sqrt{\sum_{a \in \mathcal{N}}\left|\frac{v_{a}}{a+1}\right|^{\frac{2 a+3}{a+2}}}$ for all $v \in \Xi\left(\Delta,\left(\frac{2 a+3}{a+2}\right)_{a=0}^{\infty}\right)$. Since for all $v, t \in\left(\Xi\left(\Delta,\left(\frac{2 a+3}{a+2}\right)_{a=0}^{\infty}\right)\right)_{\psi}$ with $v_{0}, t_{0} \in\left(-\infty, \frac{1}{3}\right)$, we have

$$
\begin{aligned}
\psi(W v-W t) & =\psi\left(\frac{1}{4}\left(v_{0}-t_{0}, v_{1}-t_{1}, v_{2}-t_{2}, \ldots\right)\right) \leq \frac{1}{\sqrt[4]{27}}\left(\psi\left(\frac{3 v}{4}\right)+\psi\left(\frac{3 t}{4}\right)\right) \\
& \leq \frac{1}{\sqrt[4]{27}}(\psi(W v-v)+\psi(W t-t)) .
\end{aligned}
$$

For all $v, t \in\left(\Xi\left(\Delta,\left(\frac{2 a+3}{a+2}\right)_{a=0}^{\infty}\right)\right)_{\psi}$ with $v_{0}, t_{0} \in\left(\frac{1}{3}, \infty\right)$, then for any $\varepsilon>0$ we have

$$
\psi(W v-W t)=0 \leq \varepsilon(\psi(W v-v)+\psi(W t-t))
$$

For all $v, t \in\left(\Xi\left(\Delta,\left(\frac{2 a+3}{a+2}\right)_{a=0}^{\infty}\right)\right)_{\psi}$ with $v_{0} \in\left(-\infty, \frac{1}{3}\right)$ and $t_{0} \in\left(\frac{1}{3}, \infty\right)$, we have

$$
\begin{aligned}
\psi(W v-W t) & =\psi\left(\frac{v}{4}\right) \leq \frac{1}{\sqrt[4]{27}} \psi\left(\frac{3 v}{4}\right)=\frac{1}{\sqrt[4]{27}} \psi(W v-v) \\
& \leq \frac{1}{\sqrt[4]{27}}(\psi(W v-v)+\psi(W t-t))
\end{aligned}
$$

Therefore, the map $W$ is a Kannan $\psi$-contraction mapping. Since $\psi$ satisfies the Fatou property, by Theorem 5.2 , the map $W$ has a unique fixed point $\frac{1}{3} e_{1} \in\left(\Xi\left(\Delta,\left(\frac{2 a+3}{a+2}\right)_{a=0}^{\infty}\right)\right)_{\psi}$. 


\section{Fixed points of Kannan pre-quasi nonexpansive mapping}

In this part, we discuss the uniform convexity (UUC 2) defined in [16] of the pre-quasi normed (sss) $(\Xi(\Delta, r))_{\psi}$.

Definition 6.1 $([1,17])$ We define the coming uniform convexity type behavior of the pre-quasi norm $\psi$ :

(1) [18] Suppose $a>0$ and $b>0$. Denote

$$
\mathbb{H}_{1}(a, b)=\left\{(v, t): v, t \in \mathfrak{A}_{\psi}, \psi(v) \leq a, \psi(t) \leq b, \psi(v-t) \geq a b\right\}
$$

When $\mathbb{H}_{1}(a, b) \neq \emptyset$, we put

$$
\mathrm{H}_{1}(a, b)=\inf \left\{1-\frac{1}{a} \psi\left(\frac{v+t}{2}\right):(v, t) \in \mathbb{H}_{1}(a, b)\right\} .
$$

When $\mathbb{H}_{1}(a, b)=\emptyset$, we put $\mathrm{H}_{1}(a, b)=1$. The function $\psi$ supports the uniform convexity (UC) if, for every $a>0$ and $b>0$, we have $\mathrm{H}_{1}(a, b)>0$. Observe that for all $a>0$, then $\mathbb{H}_{1}(a, b) \neq \emptyset$ for very small $b>0$.

(2) [16] The function $\psi$ provides (UUC) if, for all $p \geq 0$ and $b>0$, there is $\beta_{1}(p, b)$ based on $p$ and $b>0$ such that

$$
\mathrm{H}_{1}(a, b)>\beta_{1}(p, b)>0 \quad \text { for } a>p \text {. }
$$

(3) [16] Assume $a>0$ and $b>0$. Denote

$$
\mathbb{H}_{2}(a, b)=\left\{(v, t): v, t \in \mathfrak{A}_{\psi}, \psi(v) \leq a, \psi(t) \leq a, \psi\left(\frac{v-t}{2}\right) \geq a b\right\}
$$

When $\mathbb{H}_{2}(a, b) \neq \emptyset$, we put

$$
\mathrm{H}_{2}(a, b)=\inf \left\{1-\frac{1}{a} \psi\left(\frac{v+t}{2}\right):(v, t) \in \mathbb{H}_{2}(a, b)\right\} .
$$

When $\mathbb{H}_{2}(a, b)=\emptyset$, we place $\mathrm{H}_{2}(a, b)=1$. The function $\psi$ supports (UC 2) if, for all $a>0$ and $b>0$, we obtain $\mathrm{H}_{2}(a, b)>0$. Observe that, for each $a>0, \mathbb{H}_{2}(a, b) \neq \emptyset$ for very small $b>0$.

(4) [16] The function $\psi$ verifies (UUC 2) if, for every $p \geq 0$ and $b>0$, there is $\beta_{2}(p, b)$ based on $p$ and $b>0$ such that

$$
\mathrm{H}_{2}(a, b)>\beta_{2}(p, b)>0 \quad \text { for } a>p .
$$

(5) [18] The function $\psi$ is strictly convex (SC) if, for all $v, t \in \mathfrak{A}_{\psi}$ so that $\psi(v)=\psi(t)$ and $\psi\left(\frac{v+t}{2}\right)=\frac{\psi(v)+\psi(t)}{2}$, we get $v=t$.

Here and after, we will need the comment: $\psi_{U}(v)=\left[\sum_{m \in U}\left|\frac{v_{m}}{m+1}\right|^{r_{m}}\right]^{\frac{1}{R}}$ for all $U \subset \mathcal{N}$ and $v \in(\Xi(\Delta, r))_{\psi}$. When $U=\emptyset$, we put $\psi_{U}(v)=0$.

Theorem 6.2 The pre-quasi norm $\psi$ on $\Xi(\Delta, r)$ is (UUC2), where $\psi(v)=$ $\left[\sum_{a=0}^{\infty}\left(\frac{\left|\sum_{z=0}^{a} \Delta v_{z}\right|}{a+1}\right)^{r_{a}}\right]^{\frac{1}{K}}$ for all $v \in \Xi(\Delta, r)$ if $\left(r_{a}\right) \in \mathfrak{R}^{\mathcal{N}} \cap \ell_{\infty}$ is increasing with $r_{0}>1$. 
Proof Assume that the setting is satisfied, $a>p \geq 0$ and $b>0$. Suppose $v, t \in \Xi(\Delta, r)$ so that

$$
\psi(v) \leq a, \psi(t) \leq a \quad \text { and } \quad \psi\left(\frac{\nu-t}{2}\right) \geq a b .
$$

From the definition of $\psi$, we have

$$
\begin{aligned}
a b & \leq \psi\left(\frac{v-t}{2}\right)=\left[\sum_{d=0}^{\infty}(d+1)^{-r_{d}}\left|\frac{v_{d}-t_{d}}{2}\right|^{r_{d}}\right]^{\frac{1}{K}} \leq\left[2^{-r_{0}} \sum_{d=0}^{\infty}(d+1)^{-r_{d}}\left|v_{d}-t_{d}\right|^{r_{d}}\right]^{\frac{1}{K}} \\
& \leq 2^{-\frac{r_{0}}{K}}\left(\left[\sum_{d=0}^{\infty}(d+1)^{-r_{d}}\left|v_{d}\right|^{r_{d}}\right]^{\frac{1}{K}}+\left[\sum_{d=0}^{\infty}(d+1)^{-r_{d}}\left|t_{d}\right|^{r_{d}}\right]^{\frac{1}{K}}\right) \\
& =2^{-\frac{r_{0}}{K}}(\psi(v)+\psi(t)) \leq 2 a,
\end{aligned}
$$

this gives $b \leq 2$. Consequently, put $P=\left\{d \in \mathcal{N}: r_{d} \geq 2\right\}$ and $Q=\left\{d \in \mathcal{N}: 1<r_{d}<2\right\}=$ $\mathcal{N} \backslash P$. For all $w \in \Xi(\Delta, r)$, one has $\psi^{K}(w)=\psi_{P}^{K}(w)+\psi_{Q}^{K}(w)$. By using the conditions, we get $\psi_{P}\left(\frac{v-t}{2}\right) \geq \frac{a b}{2}$ or $\psi_{Q}\left(\frac{v-t}{2}\right) \geq \frac{a b}{2}$. Assume first $\psi_{P}\left(\frac{v-t}{2}\right) \geq \frac{a b}{2}$. Using Lemma 2.11, we obtain

$$
\psi_{P}^{K}\left(\frac{v+t}{2}\right)+\psi_{P}^{K}\left(\frac{v-t}{2}\right) \leq \frac{\psi_{P}^{K}(v)+\psi_{P}^{K}(t)}{2}
$$

this explains

$$
\psi_{P}^{K}\left(\frac{v+t}{2}\right) \leq \frac{\psi_{P}^{K}(v)+\psi_{P}^{K}(t)}{2}-\left(\frac{a b}{2}\right)^{K} .
$$

As

$$
\psi_{Q}^{K}\left(\frac{v+t}{2}\right) \leq \frac{\psi_{Q}^{K}(v)+\psi_{Q}^{K}(t)}{2}
$$

one can see

$$
\psi^{K}\left(\frac{v+t}{2}\right) \leq \frac{\psi^{K}(v)+\psi^{K}(t)}{2}-\left(\frac{a b}{2}\right)^{K} \leq a^{K}\left(1-\left(\frac{b}{2}\right)^{K}\right) .
$$

This gives

$$
\psi\left(\frac{v+t}{2}\right) \leq a\left(1-\left(\frac{b}{2}\right)^{K}\right)^{\frac{1}{K}} .
$$

Next, suppose $\psi_{Q}\left(\frac{v-t}{2}\right) \geq \frac{a b}{2}$. Set $B=\frac{b}{4}$,

$$
Q_{1}=\left\{d \in Q:\left|v_{d}-t_{d}\right| \leq B\left(\left|v_{d}\right|+\left|t_{d}\right|\right)\right\} \quad \text { and } \quad Q_{2}=Q \backslash Q_{1} \text {. }
$$

Since the power function is convex and $B \leq 1$,

$$
\psi_{Q_{1}}^{K}\left(\frac{v-t}{2}\right) \leq \sum_{d \in Q_{1}} B^{r_{d}}(d+1)^{-r_{d}}\left|\frac{\left|v_{d}\right|+\left|t_{d}\right|}{2}\right|^{r_{d}} \leq\left(\frac{B}{2}\right)^{r_{0}}\left(\psi_{Q_{1}}^{K}(v)+\psi_{Q_{1}}^{K}(t)\right)
$$




$$
\leq \frac{B}{2}\left(\psi_{Q}^{K}(v)+\psi_{Q}^{K}(t)\right) \leq B a^{K} .
$$

Since $\psi_{Q}\left(\frac{\nu-t}{2}\right) \geq \frac{a b}{2}$, we get

$$
\psi_{Q_{2}}^{K}\left(\frac{v-t}{2}\right)=\psi_{Q}^{K}\left(\frac{v-t}{2}\right)-\psi_{Q_{1}}^{K}\left(\frac{v-t}{2}\right) \geq a^{K}\left(\left(\frac{b}{2}\right)^{K}-\left(\frac{b}{4}\right)^{K}\right) .
$$

For any $d \in Q_{2}$, we have

$$
r_{0}-1 \leq r_{d}\left(r_{d}-1\right) \quad \text { and } \quad B \leq B^{2-r_{d}} \leq\left|\frac{v_{d}-t_{d}}{\left|v_{d}\right|+\left|t_{d}\right|}\right|^{2-r_{d}} .
$$

By Lemma 2.11, we have that

$$
\begin{aligned}
& (d+1)^{-r_{d}}\left|\frac{v_{d}+t_{d}}{2}\right|^{r_{d}}+\frac{\left(r_{0}-1\right) B}{2}(d+1)^{-r_{d}}\left|\frac{v_{d}-t_{d}}{2}\right|^{r_{d}} \\
& \leq \frac{1}{2}\left((d+1)^{-r_{d}}\left|v_{d}\right|^{r_{d}}+(d+1)^{-r_{d}}\left|t_{d}\right|^{r_{d}}\right) .
\end{aligned}
$$

Hence

$$
\psi_{\mathrm{Q}_{2}}^{K}\left(\frac{v+t}{2}\right)+\frac{\left(r_{0}-1\right) B}{2} \psi_{\mathrm{Q}_{2}}^{K}\left(\frac{v-t}{2}\right) \leq \frac{\psi_{\mathrm{Q}_{2}}^{K}(v)+\psi_{\mathrm{Q}_{2}}^{K}(t)}{2},
$$

this leads to

$$
\psi_{\mathrm{Q}_{2}}^{K}\left(\frac{v+t}{2}\right) \leq \frac{\psi_{\mathrm{Q}_{2}}^{K}(v)+\psi_{\mathrm{Q}_{2}}^{K}(t)}{2}-\frac{\left(r_{0}-1\right)}{2}\left(\frac{b}{4}\right)^{1+K} a^{K}\left(2^{K}-1\right) .
$$

Since

$$
\psi_{Q_{1}}^{K}\left(\frac{v+t}{2}\right) \leq \frac{\psi_{Q_{1}}^{K}(v)+\psi_{Q_{1}}^{K}(t)}{2}
$$

one has

$$
\psi_{Q}^{K}\left(\frac{v+t}{2}\right) \leq \frac{\psi_{Q}^{K}(v)+\psi_{Q}^{K}(t)}{2}-\frac{\left(r_{0}-1\right)}{2}\left(\frac{b}{4}\right)^{1+K} a^{K}\left(2^{K}-1\right) .
$$

Since

$$
\psi_{P}^{K}\left(\frac{v+t}{2}\right) \leq \frac{\psi_{P}^{K}(v)+\psi_{P}^{K}(t)}{2},
$$

we obtain

$$
\psi\left(\frac{v+t}{2}\right) \leq a\left[1-\frac{\left(r_{0}-1\right)}{2}\left(\frac{b}{4}\right)^{1+K}\left(2^{K}-1\right)\right]^{\frac{1}{K}} .
$$

It is clear that

$$
1<r_{0} \leq K<2^{K} \quad \Rightarrow \quad 0<\frac{r_{0}-1}{2^{K}-1}<1 .
$$


If we put

$$
\beta_{2}(p, b)=\min \left(1-\left(1-\left(\frac{b}{2}\right)^{K}\right)^{\frac{1}{K}}, 1-\left[1-\frac{\left(r_{0}-1\right)}{2}\left(\frac{b}{4}\right)^{1+K}\left(2^{K}-1\right)\right]^{\frac{1}{K}}\right) .
$$

Therefore, we have $\mathrm{H}_{2}(a, b)>\beta_{2}(p, b)>0$, we conclude that $\psi$ is (UUC2).

In this part, we investigate the property (R) of the pre-quasi normed (sss) $(\Xi(\Delta, r))_{\psi}$.

Theorem 6.3 If $\left(r_{a}\right) \in \mathfrak{R}^{+\mathcal{N}} \cap \ell_{\infty}$ is increasing with $r_{0}>1$, then:

(1) The space $(\Xi(\Delta, r))_{\psi}$ is a pre-quasi Banach (sss), where $\psi(v)=\left[\sum_{a=0}^{\infty}\left(\frac{\left|\sum_{z=0}^{a} \Delta v_{z}\right|}{a+1}\right)^{r_{a}}\right]^{\frac{1}{K}}$ for all $v \in \Xi(\Delta, r)$.

(2) Assume that $\Lambda$ is a nonempty $\psi$-closed and $\psi$-convex subset of $(\Xi(\Delta, r))_{\psi}$. Suppose that $v \in(\Xi(\Delta, r))_{\psi}$ is such that

$$
d_{\psi}(v, \Lambda)=\inf \{\psi(v-t): t \in \Lambda\}<\infty
$$

Then there is unique $\lambda \in \Lambda$ such that $d_{\psi}(v, \Lambda)=\psi(v-\lambda)$.

(3) $(\Xi(\Delta, r))_{\psi}$ satisfies the property $(R)$, i.e., for all decreasing sequence $\left\{\Lambda_{j}\right\}_{j \in \mathcal{N}}$ of $\psi$-closed and $\psi$-convex nonempty subsets of $(\Xi(\Delta, r))_{\psi}$ so that $\sup _{j \in \mathcal{N}} d_{\psi}\left(v, \Lambda_{j}\right)<\infty$ for some $v \in(\Xi(\Delta, r))_{\psi}$, hence we have $\bigcap_{j \in \mathcal{N}} \Lambda_{j} \neq \emptyset$.

Proof Let the conditions be satisfied. The proof of (1) follows from Theorem 4.3. To show (2), let $v \notin \Lambda$ as $\Lambda$ is $\psi$-closed. Hence, we get $A:=d_{\psi}(v, \Lambda)>0$. Therefore, for all $p \in \mathcal{N}$, there is $t_{p} \in \Lambda$ such that $\psi\left(v-t_{p}\right)<A\left(1+\frac{1}{p}\right)$. Suppose $\left\{\frac{t_{p}}{2}\right\}$ is not $\psi$-Cauchy. Hence there is a subsequence $\left\{\frac{t_{f(p)}}{2}\right\}$ and $b_{0}>0$ such that $\psi\left(\frac{t_{f(p)}-t_{f(q)}}{2}\right) \geq b_{0}$ for all $p>q \geq 0$. Furthermore, one has $\mathrm{H}_{2}\left(A\left(1+\frac{1}{p}\right), \frac{b_{0}}{2 A}\right)>\xi:=\beta_{2}\left(A\left(1+\frac{1}{p}\right), \frac{b_{0}}{2 A}\right)>0$ for each $p \in \mathcal{N}$. As

$$
\max \left(f\left(v-t_{f(p)}\right), f\left(v-t_{f(q)}\right)\right) \leq A\left(1+\frac{1}{f(q)}\right)
$$

and

$$
\psi\left(\frac{t_{f(p)}-t_{f(q)}}{2}\right) \geq b_{0} \geq A\left(1+\frac{1}{f(q)}\right) \frac{b_{0}}{2 A}
$$

for every $p>q \geq 0$, one can see

$$
\psi\left(v-\frac{t_{f(p)}+t_{f(q)}}{2}\right) \leq A\left(1+\frac{1}{f(q)}\right)(1-\xi)
$$

So

$$
A=d_{\psi}(v, \Lambda) \leq A\left(1+\frac{1}{f(q)}\right)(1-\xi)
$$

for any $q \in \mathcal{N}$. If we let $q \rightarrow \infty$, we get

$$
0<A \leq A\left(1+\frac{1}{f(q)}\right)(1-\xi)<A,
$$


this gives a contradiction. Hence, $\left\{\frac{t_{p}}{2}\right\}$ is $\psi$-Cauchy. As $(\Xi(\Delta, r))_{\psi}$ is $\psi$-complete, so $\left\{\frac{t_{p}}{2}\right\}$ $\psi$-converges to some $t$. For all $q \in \mathcal{N}$, we have the sequence $\left\{\frac{t_{p}+t_{q}}{2}\right\} \psi$-converges to $t+\frac{t_{q}}{2}$. As $\Lambda$ is $\psi$-closed and $\psi$-convex, we obtain $t+\frac{t_{q}}{2} \in \Lambda$. Surely $t+\frac{t_{q}}{2} \psi$-converges to $2 t$, this gives $2 t \in \Lambda$. By putting $\lambda=2 t$ and using Theorem 4.5 , since $\psi$ satisfies the Fatou property, one has

$$
\begin{aligned}
d_{\psi}(v, \Lambda) & \leq \psi(v-\lambda) \leq \sup _{i} \inf _{q \geq i} \psi\left(v-\left(t+\frac{t_{q}}{2}\right)\right) \leq \sup _{i} \inf _{q \geq i} \operatorname{supinf}_{i} \operatorname{in}_{p \geq i} \psi\left(v-\frac{t_{p}+t_{q}}{2}\right) \\
& \leq \frac{1}{2} \sup _{i} \inf _{q \geq i} \sup _{i} \inf _{p \geq i}\left[\psi\left(v-t_{p}\right)+\psi\left(v-t_{q}\right)\right]=d_{\psi}(v, \Lambda) .
\end{aligned}
$$

Therefore, $\psi(v-\lambda)=d_{\psi}(v, \Lambda)$. Since the function $\psi$ is (UUC2), hence it is (SC), which implies the uniqueness of $\lambda$. To show (3), let $v \notin \Lambda_{p_{0}}$ for some $p_{0} \in \mathcal{N}$. Since $\left(d_{\psi}\left(v, \Lambda_{p}\right)\right)_{p \in \mathcal{N}} \in$ $\ell_{\infty}$ is increasing, put $\lim _{p \rightarrow \infty} d_{\psi}\left(v, \Lambda_{p}\right)=A$ if $A>0$. Else $v \in \Lambda_{p}$ for all $p \in \mathcal{N}$. From (2), there is one point $t_{p} \in \Lambda_{p}$ such that $d_{\psi}\left(v, \Lambda_{p}\right)=\psi\left(v-t_{p}\right)$ for every $p \in \mathcal{N}$. A consistent proof will prove that $\left\{\frac{t_{p}}{2}\right\} \psi$-converges to some $t \in(\Xi(\Delta, r))_{\psi}$. As $\left\{\Lambda_{p}\right\}$ is $\psi$-convex, decreasing, and $\psi$-closed, one has $2 t \in \bigcap_{p \in \mathcal{N}} \Lambda_{p}$.

In this part, we explain the $\psi$-normal structure property of the pre-quasi normed (sss) $(\Xi(\Delta, r))_{\psi}$.

Definition 6.4 $(\Xi(\Delta, r))_{\psi}$ satisfies the $\psi$-normal structure property if, for all nonempty $\psi$-bounded, $\psi$-convex, and $\psi$-closed subset $\Lambda$ of $(\Xi(\Delta, r))_{\psi}$ not decreased to one point, there is $v \in \Lambda$ such that

$$
\sup _{t \in \Lambda} \psi(v-t)<\delta_{\psi}(\Lambda):=\sup \{\psi(v-t): v, t \in \Lambda\}<\infty .
$$

Theorem 6.5 If $\left(r_{a}\right) \in \mathfrak{R}^{+\mathcal{N}} \cap \ell_{\infty}$ is increasing with $r_{0}>1$, then $(\Xi(\Delta, r))_{\psi}$ has the $\psi$ normal structure property, where $\psi(v)=\left[\sum_{a=0}^{\infty}\left(\frac{\left|\sum_{z=0}^{a} \Delta v_{z}\right|}{a+1}\right)^{r_{a}}\right]^{\frac{1}{K}}$ for every $v \in \Xi(\Delta, r)$.

Proof Let the conditions be satisfied. Theorem 6.2 gives that $\psi$ is (UUC2). Suppose that $\Lambda$ is a $\psi$-bounded, $\psi$-convex, and $\psi$-closed subset of $(\Xi(\Delta, r))_{\psi}$ not decreasing to the unique point. Therefore, $\delta_{\psi}(\Lambda)>0$. Put $A=\delta_{\psi}(\Lambda)$. Assume $v, t \in \Lambda$ so that $v \neq t$. So $\psi\left(\frac{v-t}{2}\right)=b>0$. For all $\lambda \in \Lambda$, one has $\psi(v-\lambda) \leq A$ and $\psi(t-\lambda) \leq A$. As $\Lambda$ is $\psi$-convex, we get $\frac{v+t}{2} \in \Lambda$. Hence

$$
\psi\left(\frac{\nu+t}{2}-\lambda\right)=\psi\left(\frac{(\nu-\lambda)+(t-\lambda)}{2}\right) \leq A\left(1-\mathrm{H}_{2}\left(A, \frac{b}{A}\right)\right)
$$

for all $\lambda \in \Lambda$. Then

$$
\sup _{\lambda \in \Lambda} \psi\left(\frac{v+t}{2}-\lambda\right) \leq A\left(1-\mathrm{H}_{2}\left(A, \frac{b}{A}\right)\right)<A=\delta_{\psi}(\Lambda) .
$$

Lemma 6.6 Let the pre-quasi normed $(s s s)(\Xi(\Delta, r))_{\psi}$ verify the $(R)$ property and the $\psi$ quasi-normal property. Suppose that $\Lambda$ is a nonempty $\psi$-bounded, $\psi$-convex, and $\psi$ closed subset of $(\Xi(\Delta, r))_{\psi}$. Assume that $W: \Lambda \rightarrow \Lambda$ is a Kannan $\psi$-nonexpansive mapping. For $a>0$, let $G_{a}=\{v \in \Lambda: \psi(v-W(v)) \leq a\} \neq \emptyset$. Put

$$
\Lambda_{a}=\cap\left\{\mathcal{B}_{\psi}(p, q): W\left(G_{a}\right) \subset \mathcal{B}_{\psi}(p, q)\right\} \cap \Lambda .
$$


Then $\Lambda_{a}$ is a nonempty, $\psi$-convex, $\psi$-closed subset of $\Lambda$ and

$$
W\left(\Lambda_{a}\right) \subset \Lambda_{a} \subset G_{a} \text { and } \delta_{\psi}\left(\Lambda_{a}\right) \leq a
$$

Proof Since $W\left(G_{a}\right) \subset \Lambda_{a}$, this gives $\Lambda_{a} \neq \emptyset$. As the $\psi$-balls are $\psi$-convex and $\psi$-closed, hence $\Lambda_{a}$ is a $\psi$-closed and $\psi$-convex subset of $\Lambda$. To show that $\Lambda_{a} \subset G_{a}$. Assume $v \in \Lambda_{a}$. If $\psi(v-W(v))=0$, one has $v \in G_{a}$. Else, suppose $\psi(v-W(v))>0$. Put

$$
p=\sup \left\{\psi(W(w)-W(v)): w \in G_{a}\right\}
$$

By using the definition of $p$, then $W\left(G_{a}\right) \subset \mathcal{B}_{\psi}(W(v), p)$. Therefore, $\Lambda_{a} \subset \mathcal{B}_{\psi}(W(v), p)$, this gives $\psi(v-W(v)) \leq p$. Assume $b>0$. So there is $w \in G_{a}$ so that $p-b \leq \psi(W(w)-W(v))$. Then

$$
\begin{aligned}
\psi(v-W(v))-b & \left.\leq p-b \leq \psi(W(w)-W(v)) \leq \frac{1}{2}(\psi(v-W(v)))+\psi(w-W(w))\right) \\
& \leq \frac{1}{2}(\psi(v-W(v))+a) .
\end{aligned}
$$

As $b$ is randomly positive, one has $\psi(v-W(v)) \leq a$, hence we have $v \in G_{a}$. As $W\left(G_{a}\right) \subset \Lambda_{a}$, one can see $W\left(\Lambda_{a}\right) \subset W\left(G_{a}\right) \subset \Lambda_{a}$, this indicates $\Lambda_{a}$ is $W$-invariant. Consequently, to show that $\delta_{\psi}\left(\Lambda_{a}\right) \leq a$. Since

$$
\psi(W(v)-W(t)) \leq \frac{1}{2}(\psi(v-W(v))+\psi(t-W(t)))
$$

for all $v, t \in G_{a}$. Let $v \in G_{a}$. Hence $W\left(G_{a}\right) \subset \mathcal{B}_{\psi}(W(v), a)$. The definition of $\Lambda_{a}$ gives $\Lambda_{a} \subset$ $\mathcal{B}_{\psi}(W(v), a)$. Therefore, $W(v) \in \bigcap_{t \in \Lambda_{a}} \mathcal{B}_{\psi}(t, a)$. So, one has $\psi(t-w) \leq a$ for all $t, w \in \Lambda_{a}$, this means $\delta_{\psi}\left(\Lambda_{a}\right) \leq a$. This completes the proof.

We study here the sufficient conditions on the pre-quasi normed (sss) $(\Xi(\Delta, r))_{\psi}$ such that the Kannan pre-quasi norm nonexpansive mapping on it has a fixed point.

Theorem 6.7 Assume that the pre-quasi normed $(s s s)(\Xi(\Delta, r))_{\psi}$ verifies the $\psi$-quasinormal property and the $(R)$ property. Suppose that $\Lambda$ is a nonempty, $\psi$-convex, $\psi$-closed, and $\psi$-bounded subset of $(\Xi(\Delta, r))_{\psi}$. Pick up $W: \Lambda \rightarrow \Lambda$ to be a Kannan $\psi$-nonexpansive mapping. Then $W$ has a fixed point.

Proof Put $a_{0}=\inf \{\psi(v-W(v)): v \in \Lambda\}$ and $a_{p}=a_{0}+\frac{1}{p}$ for all $p \geq 1$. From the definition of $a_{0}$, one has $G_{a_{p}}=\left\{v \in \Lambda: \psi(v-W(v)) \leq a_{p}\right\} \neq \emptyset$ for every $p \geq 1$. Let $\Lambda_{a_{p}}$ be indicated as in Lemma 6.6. Evidently, $\left\{\Lambda_{a_{p}}\right\}$ is a decreasing sequence of nonempty $\psi$-bounded, $\psi$-closed, and $\psi$-convex subsets of $\Lambda$. The property (R) explains that $\Lambda_{\infty}=\bigcap_{p \geq 1} \Lambda_{a_{p}} \neq \emptyset$. Assume $v \in \Lambda_{\infty}$, one can see $\psi(v-W(v)) \leq a_{p}$ for all $p \geq 1$. Let $p \rightarrow \infty$, we have $\psi(v-W(v)) \leq a_{0}$, this gives $\psi(v-W(v))=a_{0}$. Therefore, $G_{a_{0}} \neq \emptyset$. We have $a_{0}=0$. Else, $a_{0}>0$ this gives that $W$ misses to have a fixed point. Let $\Lambda_{a_{0}}$ be as defined in Lemma 6.6. As $W$ misses to have a fixed point and $\Lambda_{a_{0}}$ is $W$-invariant, so $\Lambda_{a_{0}}$ has more than one point. This implies $\delta_{\psi}\left(\Lambda_{a_{0}}\right)>0$. By the $\psi$-quasi-normal property, there is $v \in \Lambda_{a_{0}}$ such that

$$
\psi(v-t)<\delta_{\psi}\left(\Lambda_{a_{0}}\right) \leq a_{0}
$$


for all $t \in \Lambda_{a_{0}}$. By Lemma 6.6, we have $\Lambda_{a_{0}} \subset G_{a_{0}}$. By definition of $\Lambda_{a_{0}}$, then $W(v) \in G_{a_{0}} \subset$ $\Lambda_{a_{0}}$. Clearly this gives

$$
\psi(v-W(v))<\delta_{\psi}\left(\Lambda_{a_{0}}\right) \leq a_{0},
$$

this contradicts the definition of $a_{0}$. So $a_{0}=0$, which explains that any point in $G_{a_{0}}$ is a fixed point of $W$, i.e., $W$ has a fixed point in $\Lambda$.

Using Theorems 6.3, 6.5, and 6.7, we obtain the following corollary.

Corollary 6.8 If $\left(r_{a}\right) \in \mathfrak{R}^{+\mathcal{N}} \cap \ell_{\infty}$ is increasing with $r_{0}>1$. Assume $\Lambda$ to be a nonempty, $\psi$ convex, $\psi$-closed, and $\psi$-bounded subset of $(\Xi(\Delta, r))_{\psi}$, where $\psi(v)=\left[\sum_{a=0}^{\infty}\left(\frac{\left|\sum_{z=0}^{a} \Delta v_{z}\right|}{a+1}\right)^{r_{a}}\right]^{\frac{1}{K}}$ for every $v \in \Xi(\Delta, r)$. Suppose that $W: \Lambda \rightarrow \Lambda$ is a Kannan $\psi$-nonexpansive mapping. Then $W$ has a fixed point.

Example 6.9 Let $W: \Lambda \rightarrow \Lambda$ with

$$
W(v)= \begin{cases}\frac{v}{4}, & \psi(v) \in[0,1), \\ \frac{v}{5}, & \psi(v) \in[1, \infty),\end{cases}
$$

where $\Lambda=\left\{v \in\left(\Xi\left(\Delta,\left(\frac{2 a+3}{a+2}\right)_{a=0}^{\infty}\right)\right)_{\psi}: v_{0}=v_{1}=0\right\}$ and $\psi(v)=\sqrt{\sum_{a \in \mathcal{N}}\left|\frac{v_{a}}{a+1}\right|^{\frac{2 a+3}{a+2}}}$ for all $v \in$ $\left(\Xi\left(\Delta,\left(\frac{2 a+3}{a+2}\right)_{a=0}^{\infty}\right)\right)_{\psi}$. From Example 5.8, the map $W$ is a Kannan $\psi$-contraction mapping. So it is a Kannan $\psi$-nonexpansive mapping. Clearly, $\Lambda$ is a nonempty, $\psi$-convex, $\psi$-closed, and $\psi$-bounded subset of $\left(\Xi\left(\Delta,\left(\frac{2 a+3}{a+2}\right)_{a=0}^{\infty}\right)\right)_{\psi}$. By Corollary 6.8, the map $W$ has one fixed point $(v=\theta)$ in $\Lambda$.

\section{Kannan contraction maps on pre-quasi operator ideal}

We investigate the existence of a fixed point of Kannan pre-quasi norm contraction mapping in the pre-quasi Banach operator ideal formed by $(\Xi(\Delta, r))_{\psi}$ and $s$-numbers.

The following theorem follows from Theorem 2.9.

Theorem 7.1 Pick up $Z$ and $M$ to be Banach spaces and $\left(r_{a}\right) \in \mathfrak{R}^{+\mathcal{N}} \cap \ell_{\infty}$ to be increasing, then $\left(S_{(\Xi(\Delta, r))_{\psi}}, \Psi\right)$, where $\Psi(W)=\psi\left(\left(s_{z}(W)\right)_{z=0}^{\infty}\right)=\left[\sum_{a=0}^{\infty}\left(\frac{\left|\sum_{z=0}^{a} \Delta s_{z}(W)\right|}{a+1}\right)^{r_{a}}\right]^{\frac{1}{K}}$ is a pre-quasi Banach operator ideal.

Theorem 7.2 Pick up $Z$ and $M$ to be Banach spaces and $\left(r_{a}\right) \in \mathfrak{R}^{+\mathcal{N}} \cap \ell_{\infty}$ to be increasing, then $\left(S_{(\Xi(\Delta, r))_{\psi}}, \Psi\right)$, where $\Psi(W)=\psi\left(\left(s_{z}(W)\right)_{z=0}^{\infty}\right)=\left[\sum_{a=0}^{\infty}\left(\frac{\left|\sum_{z=0}^{a} \Delta s_{z}(W)\right|}{a+1}\right)^{r_{a}}\right]^{\frac{1}{K}}$ is a pre-quasi closed operator ideal.

Proof Assume $W_{q} \in S_{(\Xi(\Delta, r))_{\psi}}(Z, M)$ for every $q \in \mathcal{N}$ and $\lim _{q \rightarrow \infty} \Psi\left(W_{q}-W\right)=0$. Since $\mathcal{L}(Z, M) \supseteq S_{(\Xi(\Delta, r))_{\psi}}(Z, M)$, we get

$$
\Psi\left(W_{q}-W\right)=\psi\left(\left(s_{a}\left(W_{q}-W\right)\right)_{a=0}^{\infty}\right) \geq \psi\left(s_{0}\left(W_{q}-W\right), 0,0,0, \ldots\right)=\left\|W_{q}-W\right\|^{r_{0}} .
$$

Hence $\left(W_{q}\right)_{q \in \mathcal{N}}$ is convergent in $\mathcal{L}(Z, M)$, i.e., $\lim _{q \rightarrow \infty}\left\|W_{q}-W\right\|=0$, and while $\left(s_{a}\left(W_{q}\right)\right)_{a=0}^{\infty} \in(\Xi(\Delta, r))_{\psi}$ for every $q \in \mathcal{N}$ and $(\Xi(\Delta, r))_{\psi}$ is a pre-modular (sss). Therefore, 
one has

$$
\begin{aligned}
\Psi(W) & =\psi\left(\left(s_{a}(W)\right)_{a=0}^{\infty}\right)=\psi\left(\left(s_{a}\left(W-W_{q}+W_{q}\right)\right)_{a=0}^{\infty}\right) \\
& \leq \psi\left(\left(s_{\left[\frac{a}{2}\right]}\left(W-W_{q}\right)\right)_{a=0}^{\infty}\right)+\psi\left(\left(s_{\left[\frac{a}{2}\right]}\left(W_{q}\right)_{a=0}^{\infty}\right)\right) \\
& \leq \psi\left(\left(\left\|W_{q}-W\right\|\right)_{a=0}^{\infty}\right)+(2)^{\frac{1}{K}} \psi\left(\left(s_{a}\left(W_{q}\right)_{a=0}^{\infty}\right)\right)<\varepsilon,
\end{aligned}
$$

we have $\left(s_{a}(W)\right)_{a=0}^{\infty} \in(\Xi(\Delta, r))_{\psi}$, then $W \in S_{(\Xi(\Delta, r))_{\psi}}(Z, M)$.

Definition 7.3 A pre-quasi norm $\Psi$ on the ideal $S_{\mathfrak{A}_{\psi}}$, where $\Psi(W)=\psi\left(\left(s_{a}(W)\right)_{a=0}^{\infty}\right)$, satisfies the Fatou property if for any sequence $\left\{W_{a}\right\}_{a \in \mathcal{N}} \subseteq S_{\mathfrak{A}_{\psi}}(Z, M)$ with $\lim _{a \rightarrow \infty} \Psi\left(W_{a}-\right.$ $W)=0$ and any $V \in S_{\mathfrak{A}_{\psi}}(Z, M)$, then

$$
\Psi(V-W) \leq \sup _{a} \inf _{i \geq a} \psi\left(V-W_{i}\right)
$$

Theorem 7.4 The pre-quasi norm $\Psi(W)=\left[\sum_{a=0}^{\infty}\left(\frac{\left|\sum_{z=0}^{a} \Delta s_{z}(W)\right|}{a+1}\right)^{r_{a}}\right]^{\frac{1}{K}}$ for all $W \in$ $S_{(\Xi(\Delta, r))_{\psi}}(Z, M)$ does not satisfy the Fatou property if $\left(r_{a}\right) \in \mathfrak{R}^{+\mathcal{N}} \cap \ell_{\infty}$ is increasing.

Proof Assume the setting to be satisfied and $\left\{W_{p}\right\}_{p \in \mathcal{N}} \subseteq S_{(\Xi(\Delta, r))_{\psi}}(Z, M)$ with $\lim _{p \rightarrow \infty} \Psi\left(W_{p}-W\right)=0$. Since the space $S_{(\Xi(\Delta, r))_{\psi}}$ is a pre-quasi closed ideal, then $W \in$ $S_{(\Xi(\Delta, r))_{\psi}}(Z, M)$. Then, for any $V \in S_{(\Xi(\Delta, r))_{\psi}}(Z, M)$, we have

$$
\begin{aligned}
\Psi(V-W) & =\left[\sum_{a=0}^{\infty}\left(\frac{\left|s_{a}(V-W)\right|}{a+1}\right)^{r_{a}}\right]^{\frac{1}{K}} \\
& \leq\left[\sum_{a=0}^{\infty}\left(\frac{\left|s_{\left[\frac{a}{2}\right]}\left(V-W_{i}\right)\right|}{a+1}\right)^{r_{a}}\right]^{\frac{1}{K}}+\left[\sum_{a=0}^{\infty}\left(\frac{\left|s_{\left[\frac{a}{2}\right]}\left(W-W_{i}\right)\right|}{a+1}\right)^{r_{a}}\right]^{\frac{1}{K}} \\
& \leq 2^{\frac{1}{K}} \sup _{p} \inf _{i \geq p}\left[\sum_{a=0}^{\infty}\left(\frac{\left|s_{a}\left(V-W_{i}\right)\right|}{a+1}\right)^{r_{a}}\right]^{\frac{1}{K}} .
\end{aligned}
$$

Hence, $\Psi$ does not satisfy the Fatou property.

Now, we give the definition of Kannan $\Psi$-Lipschitzian mapping in the pre-quasi operator ideal.

Definition 7.5 For the pre-quasi norm $\Psi$ on the ideal $S_{\mathfrak{A}_{\psi}}$, where $\Psi(W)=\psi\left(\left(s_{a}(W)\right)_{a=0}^{\infty}\right)$. An operator $G: S_{\mathfrak{A}_{\psi}}(Z, M) \rightarrow S_{\mathfrak{A}_{\psi}}(Z, M)$ is called a Kannan $\Psi$-Lipschitzian, if there is $\xi \geq 0$ such that

$$
\Psi(G W-G A) \leq \xi(\Psi(G W-W)+\Psi(G A-A))
$$

for all $W, A \in S_{\mathfrak{A}_{\psi}}(Z, M)$. An operator $G$ is called:

(1) Kannan $\Psi$-contraction if $\xi \in\left[0, \frac{1}{2}\right)$.

(2) Kannan $\Psi$-nonexpansive if $\xi=\frac{1}{2}$. 
Definition 7.6 For the pre-quasi norm $\Psi$ on the ideal $S_{\mathfrak{A}_{\psi}}$, where $\Psi(W)=\psi\left(\left(s_{a}(W)\right)_{a=0}^{\infty}\right)$, $G: S_{\mathfrak{A}_{\psi}}(Z, M) \rightarrow S_{\mathfrak{A}_{\psi}}(Z, M)$ and $B \in S_{\mathfrak{A}_{\psi}}(Z, M)$. The operator $G$ is called $\Psi$-sequentially continuous at $B$ if and only if, when $\lim _{p \rightarrow \infty} \Psi\left(W_{p}-B\right)=0$, then $\lim _{p \rightarrow \infty} \Psi\left(G W_{p}-G B\right)=0$.

Theorem 7.7 Let $\left(r_{a}\right) \in \mathfrak{R}^{+\mathcal{N}} \cap \ell_{\infty}$ be increasing and $G: S_{(\Xi(\Delta, r))_{\psi}}(Z, M) \rightarrow$ $S_{(\Xi(\Delta, r))_{\psi}}(Z, M)$, where $\Psi(W)=\left[\sum_{a=0}^{\infty}\left(\frac{\left|\sum_{z=0}^{a} \Delta s_{z}(W)\right|}{a+1}\right)^{r}\right]^{\frac{1}{K}}$ for all $W \in S_{(\Xi(\Delta, r))_{\psi}}(Z, M)$. The point $A \in S_{(\Xi(\Delta, r))_{\psi}}(Z, M)$ is the unique fixed point of $G$ if the following conditions are satisfied:

(a) $G$ is a Kannan $\Psi$-contraction mapping;

(b) $G$ is $\Psi$-sequentially continuous at a point $A \in S_{(\Xi(\Delta, r))_{\psi}}(Z, M)$;

(c) There is $B \in S_{(\Xi(\Delta, r))_{\psi}}(Z, M)$ such that the sequence of iterates $\left\{G^{p} B\right\}$ has a subsequence $\left\{G^{p_{i}} B\right\}$ converging to $A$.

Proof Let the conditions be verified. If $A$ is not a fixed point of $G$, then $G A \neq A$. From conditions (b) and (c), we have

$$
\lim _{p_{i} \rightarrow \infty} \Psi\left(G^{p_{i}} B-A\right)=0 \text { and } \lim _{p_{i} \rightarrow \infty} \Psi\left(G^{p_{i}+1} B-G A\right)=0 .
$$

Since $G$ is a Kannan $\Psi$-contraction mapping, one can see

$$
\begin{aligned}
0 & <\Psi(G A-A)=\Psi\left(\left(G A-G^{p_{i}+1} B\right)+\left(G^{p_{i}} B-A\right)+\left(G^{p_{i}+1} B-G^{p_{i}} B\right)\right) \\
& \leq 2^{\frac{1}{K}} \Psi\left(G^{p_{i}+1} B-G A\right)+2^{\frac{2}{K}} \Psi\left(G^{p_{i}} B-A\right)+2^{\frac{2}{K}} \xi\left(\frac{\xi}{1-\xi}\right)^{p_{i}-1} \Psi(G B-B) .
\end{aligned}
$$

As $p_{i} \rightarrow \infty$, we have a contradiction. Therefore, $A$ is a fixed point of $G$. To show that the fixed point $A$ is unique, let us have two different fixed points $A, D \in S_{(\Xi(\Delta, r))_{\psi}}(Z, M)$ of $G$. Hence, we obtain

$$
\Psi(A-D) \leq \Psi(G A-G D) \leq \xi(\Psi(G A-A)+\Psi(G D-D))=0 .
$$

Therefore, $A=D$.

Example 7.8 Let $Z$ and $M$ be Banach spaces, $G: S_{\left(\Xi\left(\Delta,\left(\frac{a+1}{a+2}\right)_{a=0}^{\infty}\right)\right)_{\psi}}(Z, M) \rightarrow$ $S_{\left(\Xi\left(\Delta,\left(\frac{a+1}{a+2}\right)_{a=0}^{\infty}\right)\right)_{\psi}}(Z, M)$, where $\Psi(W)=\sum_{a=0}^{\infty}\left(\frac{\left|\sum_{z=0}^{a} \Delta s_{z}(W)\right|}{a+1}\right)^{\frac{a+1}{a+2}}$ for every $W \in$ $S_{\left(\Xi\left(\Delta,\left(\frac{a+1}{a+2}\right)_{a=0}^{\infty}\right)\right)_{\psi}}(Z, M)$ and

$$
G(W)= \begin{cases}\frac{W}{26}, & \Psi(W) \in[0,1), \\ \frac{W}{37}, & \Psi(W) \in[1, \infty)\end{cases}
$$

Since for all $W_{1}, W_{2} \in S_{\left(\Xi\left(\Delta,\left(\frac{a+1}{a+2}\right)_{a=0}^{\infty}\right)\right)_{\psi}}$ with $\Psi\left(W_{1}\right), \Psi\left(W_{2}\right) \in[0,1)$, we have

$$
\begin{aligned}
\Psi\left(G W_{1}-G W_{2}\right) & =\Psi\left(\frac{W_{1}}{26}-\frac{W_{2}}{26}\right) \leq \frac{2}{5}\left(\Psi\left(\frac{25 W_{1}}{26}\right)+\Psi\left(\frac{25 W_{2}}{26}\right)\right) \\
& =\frac{2}{5}\left(\Psi\left(G W_{1}-W_{1}\right)+\Psi\left(G W_{2}-W_{2}\right)\right) .
\end{aligned}
$$


For all $W_{1}, W_{2} \in S_{\left(\Xi\left(\Delta,\left(\frac{a+1}{a+2}\right)_{a=0}^{\infty}\right)\right)_{\psi}}$ with $\Psi\left(W_{1}\right), \Psi\left(W_{2}\right) \in[1, \infty)$, we have

$$
\begin{aligned}
\Psi\left(G W_{1}-G W_{2}\right) & =\Psi\left(\frac{W_{1}}{37}-\frac{W_{2}}{37}\right) \leq \frac{1}{3}\left(\Psi\left(\frac{36 W_{1}}{37}\right)+\Psi\left(\frac{36 W_{2}}{37}\right)\right) \\
& =\frac{1}{3}\left(\Psi\left(G W_{1}-W_{1}\right)+\Psi\left(G W_{2}-W_{2}\right)\right) .
\end{aligned}
$$

For all $W_{1}, W_{2} \in S_{\left(\Xi\left(\Delta,\left(\frac{a+1}{a+2}\right)_{a=0}^{\infty}\right)\right)_{\psi}}$ with $\Psi\left(W_{1}\right) \in[0,1)$ and $\Psi\left(W_{2}\right) \in[1, \infty)$, we have

$$
\begin{aligned}
\Psi\left(G W_{1}-G W_{2}\right) & =\Psi\left(\frac{W_{1}}{26}-\frac{W_{2}}{37}\right) \leq \frac{2}{5} \Psi\left(\frac{25 W_{1}}{26}\right)+\frac{1}{3} \Psi\left(\frac{36 W_{2}}{37}\right) \\
& \leq \frac{2}{5}\left(\Psi\left(\frac{25 W_{1}}{26}\right)+\Psi\left(\frac{36 W_{2}}{37}\right)\right) \\
& =\frac{2}{5}\left(\Psi\left(G W_{1}-W_{1}\right)+\Psi\left(G W_{2}-W_{2}\right)\right) .
\end{aligned}
$$

Therefore, the map $W$ is a Kannan $\Psi$-contraction mapping and

$$
G^{p}(W)= \begin{cases}\frac{W}{26^{p}}, & \Psi(W) \in[0,1) \\ \frac{W}{37^{p}}, & \Psi(W) \in[1, \infty)\end{cases}
$$

It is clear that $G$ is $\Psi$-sequentially continuous at the zero operator $\Theta \in S_{\left(\Xi\left(\Delta,\left(\frac{a+1}{a+2}\right)_{a=0}^{\infty}\right)\right)_{\psi}}$ and $\left\{G^{p} W\right\}$ has a subsequence $\left\{G^{p_{i}} W\right\}$ converging to $\Theta$. By Theorem 7.7, the zero operator $\Theta \in S_{\left(\Xi\left(\Delta,\left(\frac{a+1}{a+2}\right)_{a=0}^{\infty}\right)\right)_{\psi}}$ is the only fixed point of $G$. Let $\left\{W^{(n)}\right\} \subseteq S_{\left(\Xi\left(\Delta,\left(\frac{a+1}{a+2}\right)_{a=0}^{\infty}\right)\right)_{\psi}}$ be such that $\lim _{n \rightarrow \infty} \Psi\left(W^{(n)}-W^{(0)}\right)=0$, where $W^{(0)} \in S_{\left(\Xi\left(\Delta,\left(\frac{a+1}{a+2}\right)_{a=0}^{\infty}\right)\right)_{\psi}}$ with $\Psi\left(W^{(0)}\right)=1$. Since the prequasi norm $\Psi$ is continuous, we have

$$
\lim _{n \rightarrow \infty} \Psi\left(G W^{(n)}-G W^{(0)}\right)=\lim _{n \rightarrow \infty} \Psi\left(\frac{W^{(n)}}{26}-\frac{W^{(0)}}{37}\right)=\Psi\left(\frac{11 W^{(0)}}{962}\right)>0 .
$$

Hence $G$ is not $\Psi$-sequentially continuous at $W^{(0)}$. So, the map $G$ is not continuous at $W^{(0)}$.

Example 7.9 Let $Z$ and $M$ be Banach spaces, $G: S_{\left(\Xi\left(\Delta,\left(\frac{2 a+1}{a+3}\right)_{a=0}^{\infty}\right)\right)_{\psi}}(Z, M) \rightarrow$ $S_{\left(\Xi\left(\Delta,\left(\frac{2 a+1}{a+3}\right)_{a=0}^{\infty}\right)\right)_{\psi}}(Z, M)$, where $\quad \Psi(W)=\sqrt{\sum_{a=0}^{\infty}\left(\frac{s_{a}(W)}{a+1}\right)^{\frac{2 a+1}{a+3}}}$ for every $W \in$ $S_{\left(\Xi\left(\Delta,\left(\frac{2 a+1}{a+3}\right)_{a=0}^{\infty}\right)\right)_{\psi}}(Z, M)$ and

$$
G(W)= \begin{cases}\frac{W}{263,170}, & \Psi(W) \in[0,1) \\ \frac{W}{263,171}, & \Psi(W) \in[1, \infty)\end{cases}
$$

Since for all $W_{1}, W_{2} \in S_{\left(\Xi\left(\Delta,\left(\frac{2 a+1}{a+3}\right)_{a=0}^{\infty}\right)\right)_{\psi}}$ with $\Psi\left(W_{1}\right), \Psi\left(W_{2}\right) \in[0,1)$, we have

$$
\begin{aligned}
\Psi\left(G W_{1}-G W_{2}\right) & =\Psi\left(\frac{W_{1}}{263,170}-\frac{W_{2}}{263,170}\right) \\
& \leq \frac{\sqrt{2}}{\sqrt[6]{263,169}}\left(\Psi\left(\frac{263,169 W_{1}}{263,170}\right)+\Psi\left(\frac{263,169 W_{2}}{263,170}\right)\right)
\end{aligned}
$$




$$
=\frac{\sqrt{2}}{\sqrt[6]{263,169}}\left(\Psi\left(G W_{1}-W_{1}\right)+\Psi\left(G W_{2}-W_{2}\right)\right) .
$$

For all $W_{1}, W_{2} \in S_{\left(\Xi\left(\Delta,\left(\frac{2 a+1}{a+3}\right)_{a=0}^{\infty}\right)\right)_{\psi}}$ with $\Psi\left(W_{1}\right), \Psi\left(W_{2}\right) \in[1, \infty)$, we have

$$
\begin{aligned}
\Psi\left(G W_{1}-G W_{2}\right) & =\Psi\left(\frac{W_{1}}{263,171}-\frac{W_{2}}{263,171}\right) \\
& \leq \frac{\sqrt{2}}{\sqrt[6]{263,170}}\left(\Psi\left(\frac{263,170 W_{1}}{263,171}\right)+\Psi\left(\frac{263,170 W_{2}}{263,171}\right)\right) \\
& =\frac{\sqrt{2}}{\sqrt[6]{263,170}}\left(\Psi\left(G W_{1}-W_{1}\right)+\Psi\left(G W_{2}-W_{2}\right)\right) .
\end{aligned}
$$

For all $W_{1}, W_{2} \in S_{\left(\Xi\left(\Delta,\left(\frac{2 a+1}{a+3}\right)_{a=0}^{\infty}\right)\right)_{\psi}}$ with $\Psi\left(W_{1}\right) \in[0,1)$ and $\Psi\left(W_{2}\right) \in[1, \infty)$, we have

$$
\begin{aligned}
\Psi\left(G W_{1}-G W_{2}\right) & =\Psi\left(\frac{W_{1}}{263,170}-\frac{W_{2}}{263,171}\right) \\
& \leq \frac{\sqrt{2}}{\sqrt[6]{263,169}} \Psi\left(\frac{263,169 W_{1}}{263,170}\right)+\frac{\sqrt{2}}{\sqrt[6]{263,170}} \Psi\left(\frac{263,170 W_{2}}{263,171}\right) \\
& \leq \frac{\sqrt{2}}{\sqrt[6]{263,169}}\left(\Psi\left(\frac{263,169 W_{1}}{263,170}\right)+\Psi\left(\frac{263,170 W_{2}}{263,171}\right)\right) \\
& =\frac{\sqrt{2}}{\sqrt[6]{263,169}}\left(\Psi\left(G W_{1}-W_{1}\right)+\Psi\left(G W_{2}-W_{2}\right)\right) .
\end{aligned}
$$

Therefore, the map $W$ is a Kannan $\Psi$-contraction mapping and

$$
G^{p}(W)= \begin{cases}\frac{W}{263,170^{p}}, & \Psi(W) \in[0,1), \\ \frac{W}{263,171^{p}}, & \Psi(W) \in[1, \infty) .\end{cases}
$$

It is clear that $G$ is $\Psi$-sequentially continuous at the zero operator $\Theta \in S_{\left(\Xi\left(\Delta,\left(\frac{2 a+1}{a+3}\right)_{a=0}^{\infty}\right)\right)_{\psi}}$ and $\left\{G^{p} W\right\}$ has a subsequence $\left\{G^{p_{i}} W\right\}$ converging to $\Theta$. By Theorem 7.7, the zero operator $\Theta \in S_{\left(\Xi\left(\Delta,\left(\frac{2 a+1}{a+3}\right)_{a=0}^{\infty}\right)\right)_{\psi}}$ is the only fixed point of $G$. Let $\left\{W^{(n)}\right\} \subseteq S_{\left(\Xi\left(\Delta,\left(\frac{2 a+1}{a+3}\right)_{a=0}^{\infty}\right)\right)_{\psi}}$ be such that $\lim _{n \rightarrow \infty} \Psi\left(W^{(n)}-W^{(0)}\right)=0$, where $W^{(0)} \in S_{\left(\Xi\left(\Delta,\left(\frac{2 a+1}{a+3}\right)_{a=0}^{\infty}\right)\right)_{\psi}}$ with $\Psi\left(W^{(0)}\right)=1$. Since the prequasi norm $\Psi$ is continuous, we have

$$
\begin{aligned}
\lim _{n \rightarrow \infty} \Psi\left(G W^{(n)}-G W^{(0)}\right) & =\lim _{n \rightarrow \infty} \Psi\left(\frac{W^{(n)}}{263,170}-\frac{W^{(0)}}{263,171}\right) \\
& =\Psi\left(\frac{W^{(0)}}{69,258,712,070}\right)>0 .
\end{aligned}
$$

Hence $G$ is not $\Psi$-sequentially continuous at $W^{(0)}$. So, the map $G$ is not continuous at $W^{(0)}$.

\section{Application to the existence of solutions of summable equations}

Summable equations such as (1) were studied by Salimi et al. [19], Agarwal et al. [20], and Hussain et al. [21]. In this section, we search for a solution to (1) in $(\Xi(\Delta, r))_{\psi}$, where 
$\left(r_{l}\right) \in \mathfrak{R}^{+\mathcal{N}}$ is increasing and $\psi(v)=\left[\sum_{a=0}^{\infty}\left(\frac{\left|\sum_{z=0}^{a} \Delta v_{z}\right|}{a+1}\right)^{r_{a}}\right]^{\frac{1}{K}}$ for all $v \in \Xi(\Delta, r)$. Consider the summable equation

$$
v_{a}=p_{a}+\sum_{m=0}^{\infty} A(a, m) f\left(m, v_{m}\right)
$$

and let $W:(\Xi(\Delta, r))_{\psi} \rightarrow(\Xi(\Delta, r))_{\psi}$ be defined by

$$
W\left(v_{a}\right)_{a \in \mathcal{N}}=\left(p_{a}+\sum_{m=0}^{\infty} A(a, m) f\left(m, v_{m}\right)\right)_{a \in \mathcal{N}} .
$$

Theorem 8.1 Summable equation (1) has a solution in $(\Xi(\Delta, r))_{\psi}$ if $A: \mathcal{N}^{2} \rightarrow \Re, f: \mathcal{N} \times$ $\mathfrak{R} \rightarrow \mathfrak{R}, p: \mathcal{N} \rightarrow \mathfrak{R}$, and for all $a \in \mathcal{N}$, there is $\xi \in\left[0, \frac{1}{2}\right)$ such that

$$
\begin{aligned}
& \left|\sum_{m \in \mathcal{N}} A(a, m)\left(f\left(m, v_{m}\right)-f\left(m, t_{m}\right)\right)\right|^{r_{a}} \\
& \quad \leq \xi^{K}\left[\left|p_{a}-v_{a}+\sum_{m=0}^{\infty} A(a, m) f\left(m, v_{m}\right)\right|^{r_{a}}+\left|p_{a}-t_{a}+\sum_{m=0}^{\infty} A(a, m) f\left(m, t_{m}\right)\right|^{r_{a}}\right] .
\end{aligned}
$$

Proof Let the conditions be verified. Consider the mapping $W:(\Xi(\Delta, r))_{\psi} \rightarrow(\Xi(\Delta, r))_{\psi}$ defined by (2). We have

$$
\begin{aligned}
\psi(W v-W t)= & {\left[\sum_{a \in \mathcal{N}}\left(\frac{\left|W v_{a}-W t_{a}\right|}{a+1}\right)^{r_{a}}\right]^{\frac{1}{K}} } \\
= & {\left[\sum_{a \in \mathcal{N}}\left(\frac{\left|\sum_{m \in \mathcal{N}} A(a, m)\left[f\left(m, v_{m}\right)-f\left(m, t_{m}\right)\right]\right|}{a+1}\right)^{r_{a}}\right]^{\frac{1}{K}} } \\
\leq & \xi\left(\left[\sum_{a \in \mathcal{N}}\left(\frac{\left|p_{a}-v_{a}+\sum_{m=0}^{\infty} A(a, m) f\left(m, v_{m}\right)\right|}{a+1}\right)^{r_{a}}\right]^{\frac{1}{K}}\right. \\
& \left.+\left[\sum_{a \in \mathcal{N}}\left(\frac{\left|p_{a}-t_{a}+\sum_{m=0}^{\infty} A(a, m) f\left(m, t_{m}\right)\right|}{a+1}\right)^{r_{a}}\right]^{\frac{1}{K}}\right) \\
= & \xi(\psi(W v-v)+\psi(W t-t)) .
\end{aligned}
$$

Then, from Theorem 5.2, we have one solution of equation $(1)$ in $(\Xi(\Delta, r))_{\psi}$.

Example 8.2 Given the sequence space $\left(\Xi\left(\Delta,\left(\frac{a+1}{a+2}\right)_{a=0}^{\infty}\right)\right)_{\psi}$, where $\psi(v)=\sum_{a \in \mathcal{N}}\left(\frac{\left|v_{a}\right|}{a+1}\right)^{\frac{a+1}{a+2}}$ for all $v \in \Xi\left(\Delta,\left(\frac{a+1}{a+2}\right)_{a=0}^{\infty}\right)$. Consider the summable equation

$$
v_{a}=e^{-(3 a+6)}+\sum_{m=0}^{\infty}(-1)^{a+m}\left(\frac{e^{\left|v_{a}\right|}}{a^{2}+m^{2}+1}\right)^{q}
$$

where $q>2$, and let $W:\left(\Xi\left(\Delta,\left(\frac{a+1}{a+2}\right)_{a=0}^{\infty}\right)\right)_{\psi} \rightarrow\left(\Xi\left(\Delta,\left(\frac{a+1}{a+2}\right)_{a=0}^{\infty}\right)\right)_{\psi}$ be defined by

$$
W\left(v_{a}\right)_{a \in \mathcal{N}}=\left(e^{-(3 a+6)}+\sum_{m=0}^{\infty}(-1)^{a+m}\left(\frac{e^{\left|v_{a}\right|}}{a^{2}+m^{2}+1}\right)^{q}\right)_{a \in \mathcal{N}} .
$$


It is easy to see that

$$
\begin{aligned}
& \left|\sum_{m=0}^{\infty}(-1)^{a}\left(\frac{e^{\left|v_{a}\right|}}{a^{2}+m^{2}+1}\right)^{q}\left((-1)^{m}-(-1)^{m}\right)\right|^{\frac{a+1}{a+2}} \\
& \leq \frac{1}{3}\left[\left|e^{-(3 a+6)}-v_{a}+\sum_{m=0}^{\infty}(-1)^{a+m}\left(\frac{e^{\left|v_{a}\right|}}{a^{2}+m^{2}+1}\right)^{q}\right|^{\frac{a+1}{a+2}}\right. \\
& \left.+\left|e^{-(3 a+6)}-t_{a}+\sum_{m=0}^{\infty}(-1)^{a+m}\left(\frac{e^{\left|t_{a}\right|}}{a^{2}+m^{2}+1}\right)^{q}\right|^{\frac{a+1}{a+2}}\right] .
\end{aligned}
$$

By Theorem 8.1, summable equation (3) has a solution in $\left(\Xi\left(\Delta,\left(\frac{a+1}{a+2}\right)_{a=0}^{\infty}\right)\right)_{\psi}$.

Example 8.3 Given the sequence space $\left(\Xi\left(\Delta,\left(\frac{2 a+1}{a+3}\right)_{a=0}^{\infty}\right)\right)_{\psi}$, where $\psi(v)=\sqrt{\sum_{a \in \mathcal{N}}\left(\frac{|v a|}{a+1}\right)^{\frac{2 a+1}{a+3}}}$ for all $v \in \Xi\left(\Delta,\left(\frac{2 a+1}{a+3}\right)_{a=0}^{\infty}\right)$. Consider the summable equation

$$
v_{a}=e^{-(3 a+6)}+\sum_{m=0}^{\infty}(-1)^{a+m}\left(\frac{v_{a}}{a^{2}+m^{2}+1}\right)^{q}
$$

where $q>2$, and let $W:\left(\Xi\left(\Delta,\left(\frac{2 a+1}{a+3}\right)_{a=0}^{\infty}\right)\right)_{\psi} \rightarrow\left(\Xi\left(\Delta,\left(\frac{2 a+1}{a+3}\right)_{a=0}^{\infty}\right)\right)_{\psi}$ be defined by

$$
W\left(v_{a}\right)_{a \in \mathcal{N}}=\left(e^{-(3 a+6)}+\sum_{m=0}^{\infty}(-1)^{a+m}\left(\frac{v_{a}}{a^{2}+m^{2}+1}\right)^{q}\right)_{a \in \mathcal{N}}
$$

It is easy to see that

$$
\begin{aligned}
& \left|\sum_{m=0}^{\infty}(-1)^{a}\left(\frac{v_{a}}{a^{2}+m^{2}+1}\right)^{q}\left((-1)^{m}-(-1)^{m}\right)\right|^{\frac{2 a+1}{a+3}} \\
& \leq \frac{1}{9}\left[\left|e^{-(3 a+6)}-v_{a}+\sum_{m=0}^{\infty}(-1)^{a+m}\left(\frac{v_{a}}{a^{2}+m^{2}+1}\right)^{q}\right|^{\frac{2 a+1}{a+3}}\right. \\
& \left.+\left|e^{-(3 a+6)}-t_{a}+\sum_{m=0}^{\infty}(-1)^{a+m}\left(\frac{t_{a}}{a^{2}+m^{2}+1}\right)^{q}\right|^{\frac{2 a+1}{a+3}}\right] .
\end{aligned}
$$

By Theorem 8.1 , summable equation (5) has a solution in $\left(\Xi\left(\Delta,\left(\frac{2 a+1}{a+3}\right)_{a=0}^{\infty}\right)\right)_{\psi}$.

Example 8.4 Given the sequence space $\left(\Xi\left(\Delta,\left(\frac{2 a+3}{a+2}\right)_{a=0}^{\infty}\right)\right)_{\psi}$, where $\psi(v)=\sqrt{\sum_{a \in \mathcal{N}}\left(\frac{\left|v_{a}\right|}{a+1}\right)^{\frac{2 a+3}{a+2}}}$ for all $v \in \Xi\left(\Delta,\left(\frac{2 a+3}{a+2}\right)_{a=0}^{\infty}\right)$. Consider the summable equation

$$
v_{a}=e^{-(3 a+6)}+\sum_{m=0}^{\infty}(-1)^{a+m}\left(\frac{v_{a}}{a^{2}+m^{2}+1}\right)^{q}
$$


with $a \geq 2$ and $q>2$, and let $W: \Lambda \rightarrow \Lambda$, where $\Lambda=\left\{v \in\left(\Xi\left(\Delta,\left(\frac{2 a+3}{a+2}\right)_{a=0}^{\infty}\right)\right)_{\psi}: v_{0}=v_{1}=0\right\}$, be defined by

$$
W\left(v_{a}\right)_{a \geq 2}=\left(e^{-(3 a+6)}+\sum_{m=0}^{\infty}(-1)^{a+m}\left(\frac{v_{a}}{a^{2}+m^{2}+1}\right)^{q}\right)_{a \geq 2} .
$$

Clearly, $\Lambda$ is a nonempty, $\psi$-convex, $\psi$-closed, and $\psi$-bounded subset of $(\Xi(\Delta$, $\left.\left.\left(\frac{2 a+3}{a+2}\right)_{a=0}^{\infty}\right)\right)_{\psi}$. It is easy to see that

$$
\begin{aligned}
& \left|\sum_{m=0}^{\infty}(-1)^{a}\left(\frac{v_{a}}{a^{2}+m^{2}+1}\right)^{q}\left((-1)^{m}-(-1)^{m}\right)\right|^{\frac{2 a+3}{a+2}} \\
& \leq \frac{1}{9}\left[\left|e^{-(3 a+6)}-v_{a}+\sum_{m=0}^{\infty}(-1)^{a+m}\left(\frac{v_{a}}{a^{2}+m^{2}+1}\right)^{q}\right|^{\frac{2 a+3}{a+2}}\right. \\
& \left.+\left|e^{-(3 a+6)}-t_{a}+\sum_{m=0}^{\infty}(-1)^{a+m}\left(\frac{t_{a}}{a^{2}+m^{2}+1}\right)^{q}\right|^{\frac{2 a+3}{a+2}}\right] .
\end{aligned}
$$

By Theorem 8.1, summable equation (7) has a solution in $\Lambda$.

\begin{abstract}
Acknowledgements
This work was funded by the University of Jeddah, Saudi Arabia, under grant No.(UJ-02-054-DR). The authors, therefore, acknowledge with thanks the University technical and financial support. Additionally, the writers wish to express their gratitude to the anonymous referees for their insightful advice and supportive comments, which resulted in a major improvement to this paper's initial draft.
\end{abstract}

\title{
Funding
}

Applicable.

\section{Availability of data and materials}

Not applicable.

\section{Ethics approval and consent to participate}

This article does not contain any studies with human participants or animals performed by any of the authors.

\section{Competing interests}

The authors declare that they have no competing interests.

\section{Authors' contributions}

All authors contributed equally to the writing of this paper. All authors read and approved the final manuscript.

\section{Author details}

'Department of Mathematics, College of Science and Arts at Khulis, University of Jeddah, Jeddah, Saudi Arabia. ${ }^{2}$ Department of Mathematics, Faculty of Science, Ain Shams University, P.O. Box 1156, Cairo, 11566, Abbassia, Egypt.

${ }^{3}$ Department of Mathematics, Academy of Engineering and Medical Sciences, Khartoum, Sudan.

\section{Publisher's Note}

Springer Nature remains neutral with regard to jurisdictional claims in published maps and institutional affiliations.

Received: 30 December 2020 Accepted: 21 May 2021 Published online: 10 June 2021

\section{References}

1. Diening, L., Harjulehto, P., Hästö, P., Ruẑiĉkka, M.: Lebesgue and Sobolev Spaces with Variable Exponents. Springer, Berlin (2011)

2. Rajagopal, K., Rużiĉka, M.: On the modeling of electrorheological materials. Mech. Res. Commun. 23, $401-407$ (1996)

3. Rużiçka, M.: Electrorheological fluids. Modeling and mathematical theory. In: Lecture Notes in Mathematics 1748. Springer, Berlin (2000)

4. Pietsch, A.: Eigenvalues and s-Numbers. Cambridge University Press, New York (1986) 
5. Faried, N., Bakery, A.A.: Small operator ideals formed by s-numbers on generalized Cesàro and Orlicz sequence spaces. J. Inequal. Appl. (2018). https://doi.org/10.1186/s13660-018-1945-y

6. Bakery, A.A., Abou Elmatty, A.R.: Pre-quasi simple Banach operator ideal generated by s-numbers. J. Funct. Spaces 2020, Article ID 9164781 (2020). https://doi.org/10.1155/2020/9164781

7. Banach, S.: Sur les opérations dans les ensembles abstraits et leurs applications. Fundam. Math. 3, 133-181 (1922)

8. Kannan, R.: Some results on fixed points- II. Am. Math. Mon. 76, 405-408 (1969)

9. Ghoncheh, S.J.H.: Some fixed point theorems for Kannan mapping in the modular spaces. Ciĕnc. Nat. 37, 462-466 (2015)

10. Bakery, A.A., Abou Elmatty, A.R.: Some properties of pre-quasi norm on Orlicz sequence space. J. Inequal. Appl. 2020, 55 (2020). https://doi.org/10.1186/s13660-020-02318-8

11. Pietsch, A.: Operator Ideals. North-Holland, Amsterdam (1980)

12. Bakery, A.A., Mohammed, M.M.: Some properties of pre-quasi operator ideal of type generalized Cesàro sequence space defined by weighted means. Open Math. 17, 1703-1715 (2019). https://doi.org/10.1515/math-2019-0135

13. Clarkson, J.A.: Uniformly convex spaces. Trans. Am. Math. Soc. 40, 396-414 (1936)

14. Sundaresan, K.: Uniform convexity of Banach spaces $\ell(\{$ pi\}). Stud. Math. 39, 227-231 (1971)

15. Altay, B., Başar, F.: Generalization of the sequence space $\ell(p)$ derived by weighted means. J. Math. Anal. Appl. 1(330), 147-185 (2007)

16. Khamsi, M.A., Kozlowski, W.M.: Fixed Point Theory in Modular Function Spaces. Birkhäuser, New York (2015)

17. Musielak, J.: Orlicz Spaces and Modular Spaces. Springer, Berlin (1983)

18. Nakano, H.: Topology of Linear Topological Spaces. Maruzen, Tokyo (1951)

19. Salimi, P., Latif, A., Hussain, N.: Modified $\alpha$ - $\psi$-contractive mappings with applications. Fixed Point Theory Appl. 2013, 151 (2013)

20. Agarwal, R.P., Hussain, N., Taoudi, M.-A.: Fixed point theorems in ordered Banach spaces and applications to nonlinear integral equations. Abstr. Appl. Anal. 2012, Article ID 245872 (2012)

21. Hussain, N., Khan, A.R., Agarwal, R.P.: Krasnosel'skii and Ky Fan type fixed point theorems in ordered Banach spaces. J. Nonlinear Convex Anal. 11(3), 475-489 (2010)

\section{Submit your manuscript to a SpringerOpen ${ }^{\circ}$ journal and benefit from:}

- Convenient online submission

- Rigorous peer review

- Open access: articles freely available online

- High visibility within the field

- Retaining the copyright to your article

Submit your next manuscript at $\boldsymbol{\text { springeropen.com }}$ 